\title{
Involvement of the Globus Pallidus in Behavioral Goal Determination and Action Specification
}

\author{
Nariko Arimura, ${ }^{1,2}$ Yoshihisa Nakayama, ${ }^{1,2}$ Tomoko Yamagata, ${ }^{1,2}$ Jun Tanji, ${ }^{2,3}$ and Eiji Hoshi ${ }^{1,2,4}$ \\ ${ }^{1}$ Frontal Lobe Function Project, Tokyo Metropolitan Institute of Medical Science, Tokyo 156-8506, Japan, ${ }^{2}$ Tamagawa University Brain Science Institute, \\ Tokyo 194-8610, Japan, ${ }^{3}$ Tohoku University Brain Science Center, Sendai 980-8577, Japan, and ${ }^{4}$ Japan Science and Technology Agency, CREST, Tokyo 102- \\ 0076, Japan
}

Multiple loop circuits interconnect the basal ganglia and the frontal cortex, and each part of the cortico-basal ganglia loops plays an essential role in neuronal computational processes underlying motor behavior. To gain deeper insight into specific functions played by each component of the loops, we compared response properties of neurons in the globus pallidus (GP) with those in the dorsal premotor cortex (PMd) and the ventrolateral and dorsolateral prefrontal cortex (vlPFC and dlPFC) while monkeys performed a behavioral task designed to include separate processes for behavioral goal determination and action selection. Initially, visual signals instructed an abstract behavioral goal, and seconds later, a choice cue to select an action was presented. When the instruction cue appeared, GP neurons started to reflect visual features as early as vlPFC neurons. Subsequently, GP neurons began to reflect goals informed by the visual signals no later than neurons in the PMd, vlPFC, and dlPFC, indicating that the GP is involved in the early determination of behavioral goals. In contrast, action specification occurred later in the GP than in the cortical areas, and the GP was not as involved in the process by which a behavioral goal was transformed into an action. Furthermore, the length of time representing behavioral goal and action was shorter in the GP than in the PMd and dlPFC, indicating that the GP may play an important role in detecting individual behavioral events. These observations elucidate the involvement of the GP in goal-directed behavior.

\section{Introduction}

Substantial structural interactions occur between the basal ganglia (BG) and the frontal cortex (Alexander et al., 1986; Flaherty and Graybiel, 1994; Inase and Tanji, 1994; Middleton and Strick, 2000; Graybiel, 2008), requiring communication through each of the cortico-BG loops during multiple computational processes essential to behavior. Among them, the BG, lateral prefrontal cortex (IPFC), and premotor cortex (PM) are shown to play a crucial role in associating visual signals with actions in a goaloriented and adaptive manner (Graybiel et al., 1994; Wise et al., 1996; Asaad et al., 1998; Hollerman et al., 2000; Wise and Murray, 2000; Inase et al., 2001; Packard and Knowlton, 2002; HadjBouziane and Boussaoud, 2003; Wallis and Miller, 2003; Amiez et al., 2006; Cisek and Kalaska, 2010; Genovesio et al., 2012). In humans, Toni et al. (2001) identified activity foci in the BG, PM, and IPFC when subjects performed hand gestures arbitrarily associated with visual objects. Toni et al. (2002) further revealed

Received April 10, 2013; revised July 7, 2013; accepted July 10, 2013.

Author contributions: N.A., Y.N., T.Y., J.T., and E.H. designed research; N.A., Y.N., T.Y., J.T., and E.H. performed research; N.A., Y.N., T.Y., J.T., and E.H. contributed unpublished reagents/analytic tools; N.A., Y.N., T.Y., J.T., and E.H. analyzed data; N.A., Y.N., T.Y., J.T., and E.H. wrote the paper.

This work was supported by CREST (E.H.), Grant-in-Aid for Young Scientists 19670004 to E.H., Grant-in-Aid for Young Scientists 22700344 to N.A., Grant-in-Aid for Scientific Research 24500397 to N.A., and Global COE Program of Tamagawa University (N.A., Y.N., T.Y.). We thank Dr. Masaki Tanaka for advice on recording neurons from the basal ganglia and T. Ogata, S. Hoffman, and Dr. L. Tremblay for technical assistance.

The authors declare no competing financial interests.

Correspondence should be addressed to Dr. Eiji Hoshi, Frontal Lobe Function Project, Tokyo Metropolitan Institute of Medical Science, Kamikitazawa 2-1-6, Setagaya-ku, Tokyo 156-8506, Japan. E-mail: hoshi-ej@igakuken.or.jp.

DOI:10.1523/JNEUROSCI.1620-13.2013

Copyright $\odot 2013$ the authors $\quad 0270-6474 / 13 / 3313639-15 \$ 15.00 / 0$ that novel visuomotor associations are established through the enhancement of cortico-basal ganglia circuits. In monkeys, Brasted and Wise (2004) showed that the neural representation of association learning between visual objects and movements progresses simultaneously in the PM and striatum. Miller and colleagues revealed that association learning in the striatum precedes that in the IPFC (Pasupathy and Miller, 2005), whereas the IPFC plays a major role in abstract categorization of visual signals for saccadic eye movements (Antzoulatos and Miller, 2011). Moreover, lesion studies established that the PM (Halsband and Passingham, 1985; Petrides, 1986; Kurata and Hoffman, 1994), lPFC (Wang et al., 2000), and the connections between the BG and PM (Nixon et al., 2004) are crucially involved in the recall and learning of the visuomotor associations. All these observations indicate that the functional associations of the BG, PM, and IPFC are crucial in the visuomotor associations for goal-directed behavior.

To this line of research, we introduced a new experimental approach to explore mechanisms of goal-directed behavior by requiring subjects to select an action that conformed to a behavioral goal instructed by a visual signal. By examining neuronal activity of monkeys performing the novel behavioral task, we found several specific roles for the dorsal premotor cortex (PMd) and IPFC (Nakayama et al., 2008; Yamagata et al., 2009, 2012). We here examined the roles played by the BG by recording neurons from the globus pallidus (GP) of the same monkeys performing the same tasks. Because GP neurons were considered to carry signals at the output stage (the internal segment) and at the intermediate stage (the external segment) of a series of informa- 
tion processing within the $\mathrm{BG}$, we considered that comparing response properties of the GP with those in the PMd and IPFC would lead to better understanding of the cortico-basal ganglia circuits. We show that GP neurons exhibit a unique spectrum of properties different from those of cortical neurons in retrieval of behavioral goals from visual signals and the specification of actions, which are two crucially important processes in goaldirected behavior.

\section{Materials and Methods}

Subjects and apparatus. The subjects were three monkeys (Macaca fuscata): two males, Monkeys 1 and 3, weighing $6.0 \mathrm{~kg}$ and $9.0 \mathrm{~kg}$, and one female, Monkey 2, weighing $5.0 \mathrm{~kg}$. We recorded neuronal activity from the left PMd of Monkey 1 and Monkey 2 and from the left GP and PFC of Monkey 1 and Monkey 3. All experiments were conducted according to the guidelines of the Tokyo Metropolitan Institute of Medical Science, Tamagawa University, and the National Institutes of Health. During the experimental sessions, each monkey sat in a chair facing a 19 inch video monitor equipped with a touch-sensitive screen. Each monkey used his or her right hand to touch the monitor and push a button in front of the chair; each monkey's head and left hand were restrained. Eye position was monitored with an infrared monitoring system (RHS-M; Applied Science Laboratories). The TEMPONET system (Reflective Computing) was used to control the behavioral task and to save data for off-line analyses at $1000 \mathrm{~Hz}$.

Behavioral task. The monkeys were trained to perform two behavioral tasks: a symbolic cue task and a direct cue task. The two tasks alternated in blocks of 30-60 trials. Trials of each task began when the monkey pushed the button and gazed at a fixation point $\left(\bigcirc ; 1.4^{\circ}\right.$ in visual angle $)$ that appeared at the center of the screen.

Symbolic cue task. This task was characterized by four behavioral phases separated in time (see Fig. 1A): (1) encoding a visual object presented as an instruction cue; (2) retrieval of a behavioral goal associated with visual cues; (3) determination of an action based on the information of the retrieved behavioral goal and the spatial position of the choice cue; and (4) execution of an action. After the monkeys maintained their gaze on the fixation point for $1200 \mathrm{~ms}$, an instruction cue $\left(11^{\circ}\right)$ was presented for $800 \mathrm{~ms}$; this cue indicated that either the left or the right target should be selected (see Fig. 1B) later in the task period, but it did not indicate the exact position of future targets. During this phase, the monkeys could determine only the relative position of the reach target (a behavioral goal) in the absence of specific information about the actual reach target because the choice cue, consisting of two potential targets, was presented at various positions on the screen (see Fig. 1C). Each of the two male monkeys (Monkey 1 and Monkey 3 ) selected a target on the right when a green circle or yellow square was presented or selected a target on the left when a red diamond or blue cross was presented (see Fig. 1B). For the female monkey (Monkey 2), a green circle and a red diamond specified the selection of the right and left targets, respectively. These cues were presented randomly. If the monkeys gazed at the fixation point for 1200 ms during the subsequent instructed delay period, a choice cue consisting of two gray squares (size of each box, $7^{\circ} ; 11^{\circ}$ apart between the centers) randomly appeared at one of six different locations on the screen (see Fig. $1 C$, from $0-1$ to $5-6$ ). At this stage, the monkeys could determine, for the first time, where to reach on the screen (an action; see Fig. 1C, T1-T5 with $\mathrm{T} 3$ at the center of the screen) by transforming the behavioral goal into an action on the basis of the choice-cue position. The positions 0 and 6 were never used as reach targets to keep the target positions identical (T1-T5) for the right and left behavioral goals. If the monkeys gazed at the choice cue for $\geq 300 \mathrm{~ms}$ at the end of the choice-cue period, which lasted for 1500-2500 ms, the color changed from gray to white, serving as the GO signal. If not, the onset of the GO signal was postponed until the fixation requirement was fulfilled. When the monkey reached for the correct target within the next $1.5 \mathrm{~s}$, the monkey received a fruit juice reward $500 \mathrm{~ms}$ after touching a correct target.

Direct cue task. This task allowed the monkey to determine an action after the instruction cue was presented (see Fig. 1D). The temporal sequence of behavioral events and the gaze requirements were identical to those of the symbolic cue task. The only difference was that the instruction cue and the set cue were both a square that appeared at one of five different locations on the screen (see Fig. 1E). Because the set cue reappeared at an identical location as the instruction cue in each trial, the monkeys could determine where to go (the action) once the instruction cue was presented.

Surgery and physiological recording. After completion of the behavioral training, aseptic surgery was performed under pentobarbital sodium anesthesia (20-25 mg/kg, i.v.) with atropine sulfate. Antibiotics and analgesics were used to prevent postsurgical infection and pain. Polycarbonate screws were implanted in the skull, and two plastic pipes were rigidly attached with acrylic resin, which were used to securely fix the head during the daily recording session. A part of the skull was removed, and a recording chamber was implanted.

To record from neurons in the cerebral cortex, we used glass-insulated Elgiloy-alloy microelectrodes $(0.5-2.0 \mathrm{M} \Omega$ at $1 \mathrm{kHz})$, which were inserted through the dura mater using a hydraulic microdrive (MO-81-S; Narishige). To record from neurons in the GP, we used glass-coated tungsten microelectrodes (2.0 $\mathrm{M} \Omega$ at $1 \mathrm{kHz}$; Alpha Omega Engineering) placed in a 23-gauge guide tube. We inserted the electrodes in the dorsoventral direction through the guide tubes, which were aimed using a grid with holes $1 \mathrm{~mm}$ apart. Electrode movement was controlled using a hydraulic microdrive (MO-97A; Narishige). Single-unit potentials were amplified with a multichannel processor and sorted using a multispike detector (MCP Plus 8 and ASD; Alpha Omega Engineering).

Recording sites were verified by examining magnetic resonance (MR) images (1.5 T; Sonata; Siemens). Tubes containing contrast agent were placed at $5 \mathrm{~mm}$ intervals in the grid used for the physiological recordings to accurately identify recording sites in the brain images.

Classification of neuronal activity. To detect neurons exhibiting taskrelated activity, we first counted the number of spikes from each neuron during successive $200 \mathrm{~ms}$ bins constituting the following five task events: instruction-cue presentation (10 bins; four before, one around, and five after the instruction-cue onset); choice-cue presentation (10 bins; four before, one around, and five after the choice-cue onset); GO-signal presentation (five bins; four before, and one around the GO-signal onset); movement execution (four bins; one before, one around, and two after the movement onset); and reward delivery (nine bins; four before, one around, and four after the delivery onset). We defined a neuron as "task related" if the discharge rate (in spikes/s) distribution significantly differed in at least one of the 10 trial types in the symbolic-cue task (see Figure $1 C$, five positions of the target on the screen, T1-T5, each of which could be associated with a left or right target; ANOVA, $p<0.001$, uncorrected). All neurons in the database (GP, $n=1002$; PMd, $n=773$; ventrolateral prefrontal cortex [vlPFC], $n=358$; dorsolateral prefrontal cortex [dlPFC], $n=791$ ) were confirmed to be task related and were sampled in $>50$ trials

For activity during each of the successive $200 \mathrm{~ms}$ bins, we initially used a one-way ANOVA $(p<0.01)$ to analyze spike-count data in each bin by examining the four visual stimuli to identify activity selective for the visual objects used as instruction cues (i.e., cue-selective activity). For neurons with cue-selective activity, we used a $t$ test with Bonferroni's correction $(\alpha=0.005)$, in which we compared activities in response to the left-instruction cue (red diamond vs blue cross) or to the rightinstruction cue (green circle vs yellow square). If the activities significantly differed in either comparison, the cue-selective neuron was judged as OBJECT selective; in other words, the OBJECT neurons were neurons responding differently to two objects instructing an identical behavioral goal.

For activity without OBJECT selectivity, we first applied a two-way ANOVA to examine selectivity for the behavioral goal (i.e., left or right side of the choice cue) or the action (i.e., the five target positions on the screen). Based on these analyses, we classified neuronal selectivity into four categories: (1) behavioral goal only (behavioral goal, $p<0.01$; action, $p \geq 0.01$; and behavioral goal by action, $p \geq 0.01$ ); (2) action only (behavioral goal, $p \geq 0.01$; action, $p<0.01$; and behavioral goal by action, $p \geq 0.01$ ); (3) selective for both (behavioral goal, $p<0.01$ and action, $p<0.01$; or behavioral goal by action, $p<0.01$ ); and (4) nonselective (behavioral goal, $p \geq 0.01$; action, $p \geq 0.01$; and behavioral goal by 
A Symbolic cue task
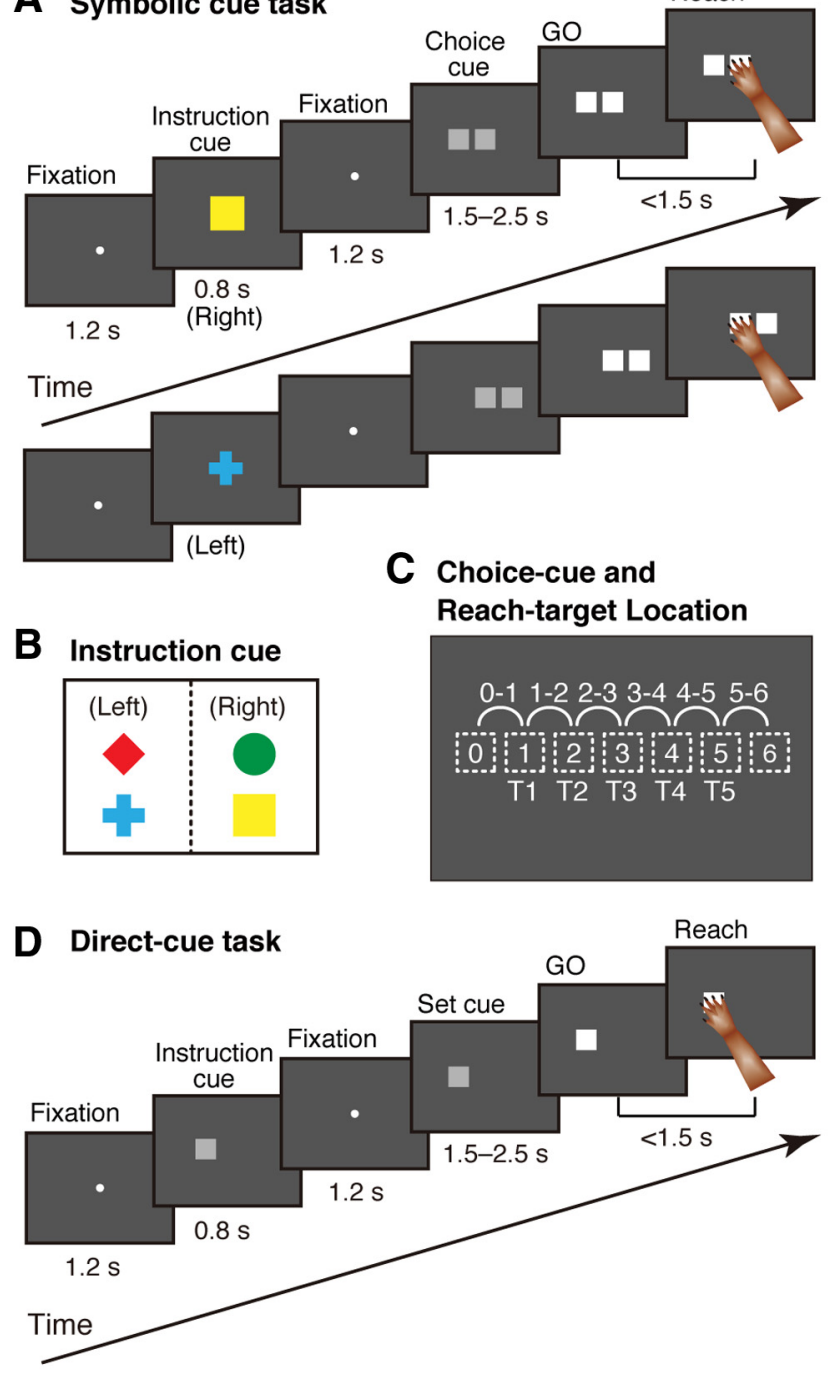

E
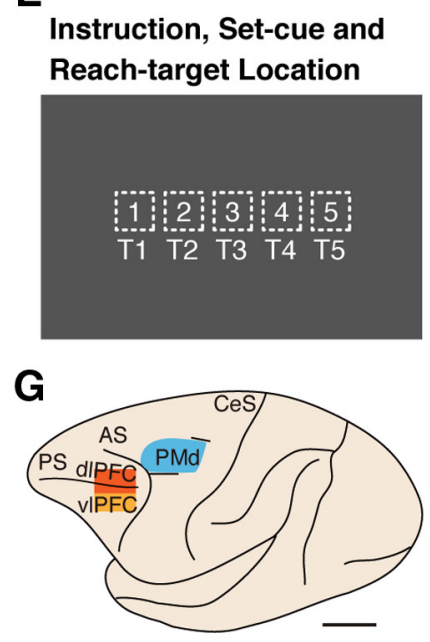

$\mathbf{F}$
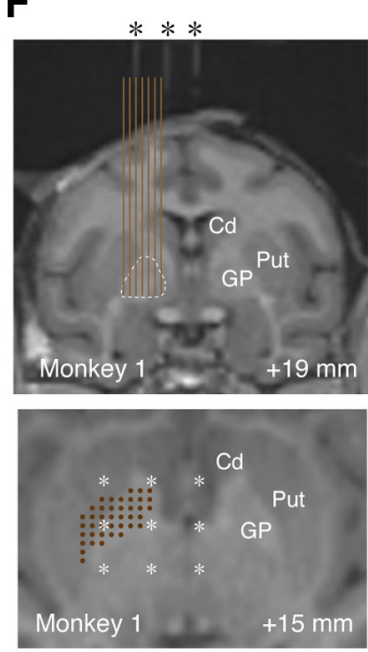

Figure 1. Behavioral tasks, instructions, cue locations, and recording sites. $\boldsymbol{A}$, Temporal sequence of behavioral events in the symbolic-cue task. $\boldsymbol{B}$, Visual signals used to designate the selection of left or right in the forthcoming choice cue. $C$, Choice-cue and reach-target locations on the screen. For the choice cue, two gray squares appeared at neighboring positions (locations $0-6$, depicted with dotted squares). The target position was selected from five potential targets (T1-T5), which were either the left or right component of the choice cue. T3 was located at the center of the screen, aligned with the center of the monkey's body. $\boldsymbol{D}$, Temporal sequence of behavioral events in the direct-cue task. $\boldsymbol{E}$, Instruction, set-cue, and reach-target locations on action, $p \geq 0.01$ ). Neuronal activity classified as both may play a role in representing the location of the choice cue or in integrating the cue location with the behavioral goal. To investigate these possibilities, we performed a second two-way ANOVA with neuronal activity classified as both to examine two main factors: the choice-cue location and the behavioral goal. For these analyses, we used the data obtained with the cues at positions 1-5 in Figure $1 C$. We excluded data from trials in which the choice cue included positions 0 and 6 (see Fig. $1 C$ ) that were never used as reach targets because it was impossible to determine whether activity integrated the choice-cue location with the behavioral goal or just represented the choice-cue location. The second analysis resulted in the identification of two classes of activity: (1) activity selective only for the choice-cue locations (choice cue, $p<0.01$; behavioral goal, $p \geq 0.01$; and choice cue by behavioral goal, $p \geq 0.01$ ); and (2) activity selective for both the choice-cue locations and the behavioral goal (choice cue, $p<0.01$, and behavioral goal, $p<0.01$; or choice cue by behavioral goal, $p<0.01$ ). The results of the two sets of ANOVAs classified activity without OBJECT selectivity into five categories: (1) selective only for the behavioral goal, (2) selective only for the action, (3) selective only for the choice-cue location, (4) selective for the choice-cue location and the behavioral goal (mixed selectivity), and (5) selective for neither.

Overall, the above analyses led to the classification of neuronal activity into six categories: (1) selective for the visual object, (2) selective only for the behavioral goal, (3) selective only for the action, (4) selective only for the choice-cue location, (5) selective for the choice-cue location and the behavioral goal (mixed selectivity), and (6) selective for none.

\section{Results}

While the monkeys performed the tasks, we recorded neuronal activity in the GP of the left hemisphere (Fig. $1 F$ ). We approached the GP vertically in the dorsoventral direction to securely record neurons in the dorsal aspect of the GP (Yoshida and Tanaka, 2009), which is interconnected with the frontal association cortex. The GP was identified based on the depth profile of microelectrode penetrations, MR images (Fig. $1 F$ ), and discriminative firing patterns. Before the electrodes entered the GP, where neurons have high tonic firing rates (DeLong, 1971), they traveled through the internal capsule or the striatum, where neuronal spikes are virtually absent or neurons have low spontaneous firing rates (Crutcher and DeLong, 1984).

We sampled the activity of every neuron that we encountered. As a result of off-line analyses, 1002 GP neurons $(n=477$ in Monkey 1 and $n=525$ in Monkey 3), 50\% of all GP neurons that we encountered during the experimental sessions were found to be "task related." Success rates during the behavioral task exceeded 95\% for each monkey; error rates were 3\% for Monkey 1, $4 \%$ for Monkey 2 , and 3\% for Monkey 3 . We analyzed the activity of GP neurons during successful trials and compared the response properties of neurons in the GP with those in the PMd and IPFC (Nakayama et al., 2008; Yamagata et al., 2009, 2012; Fig. $1 G)$. In each area, we recorded neurons from two monkeys; neurons in GP, dlPFC, and vlPFC were recorded from Monkey 1 and

$\leftarrow$

the screen in the direct-cue task. The position was selected from five potential targets (T1-T5) as in the symbolic cue task (shown in C). $\boldsymbol{F}$, Localization of recording sites with magnetic resonance images from Monkey 1 . Monkeys were scanned with tubes containing contrast agent (asterisks) in place in the grid (at intervals of $5 \mathrm{~mm}$ ). Top, Coronal plane. The recording sites (at intervals of $1 \mathrm{~mm}$, brown vertical lines) were verified based on grid coordinates with holes every $1 \mathrm{~mm}$ and on electrode depth measurements. The GP is depicted with dotted lines. Bottom, Position of recording sites (brown dots) and tubes containing contrast agent (white asterisk) that projected onto a horizontal plane through the recording site. Numbers indicate the distance from the interaural line in the rostrocaudal (top) and dorsoventral axis (bottom). Cd, Caudate; Put, putamen. G, Cortical map of the recorded areas. Neurons from the PMd (blue), dIPFC (red), and vIPFC (orange) were analyzed. PS, Principal sulcus; AS, arcuate sulcus; CeS, central sulcus. Scale bar, $10 \mathrm{~mm}$. 
Monkey 3, and neurons in PMd were from Monkey 1 and Monkey 2. Monkey 2 was able to perform the task relying only on the two instruction objects (i.e., a red diamond and a green circle). Thus, we could analyze the object feature selectivity in PMd neurons recorded only from Monkey 1 . However, we consider that this does not affect the main conclusions of the present study because the PMd neurons are shown to be generally not selective for the visual object features (Wallis and Miller, 2003) as verified in Monkey 1 in the present study (Nakayama et al., 2008; Yamagata et al., 2012).

Based on the reconstruction of the recording sites, it was found that 808 task-related neurons were in the GPe and 194 task-related neurons were in the GPi. Examination of temporal profiles of the task-selective neurons and of the timing of the selectivity development, whose results are described in detail in the following sections, revealed that response properties of GPe and GPi neurons were not significantly different. Thus, we show the results of total GP neurons together, including the external and internal segments of the GP, unless otherwise stated.

\section{Examples of neuronal activity in the GP in response to the} instruction cue and choice cue

After instruction-cue presentation, the monkeys could determine the behavioral goal based on the visual object. Figure 2 illustrates two examples of neuronal responses observed in the GP. The first type of activity reflected the visual features of the object presented as an instruction cue. The GP neuron in Figure $2 A$ showed a transient decrease in activity after a red diamond appeared, whereas its activity increased after the appearance of a blue cross. The second type of responses to the instruction cue is shown in Figure $2 B$. In this example, the activity transiently decreased when a red diamond or blue cross was presented. As explained in the following section, we interpreted the second type of response as primarily indicating the behavioral goal of reaching to the left target in the example displayed in Figure $2 B$.

In the task period after the appearance of the choice cue, the monkeys could specify the action (i.e., the location of the correct reach target on the screen) based on the behavioral goal and the choice-cue location. We found that a subset of GP neurons began to reflect the action after choice-cue onset. The neuron presented in Figure 3 showed a transient increase in neuronal activity when the choice cue was given, and the activity depended on the position of the correct reach target; the activity was greater when the target to be reached was in the center (T3) or on the right side (T4 and $\mathrm{T} 5$ ) of the screen regardless of the behavioral goals.

The three examples of neurons described above prompted us to look into the possibility that the GP is involved in progressive stages of the perception-action transformation, including behavioral goal determination and action specification. Thus, in the present study, we focused on neuronal activity from the instruction-cue onset until the GO-signal onset. Neuronal activity after the GO-signal onset is a subject of another report.

\section{Population analysis of GP neuron activity after} instruction-cue presentation

To examine the time course of population responses of GP neurons to the instruction cue and the choice cue and their relation to behavioral factors, we performed an ANOVA on the activity of individual neurons in each $200 \mathrm{~ms}$ bin beginning from the trial start and ending at the GO signal onset (i.e., from $900 \mathrm{~ms}$ before the onset of the instruction cue to $100 \mathrm{~ms}$ after the onset of the GO signal). We then constructed the time-dependent distribution histograms of neurons exhibiting selectivity to each of the

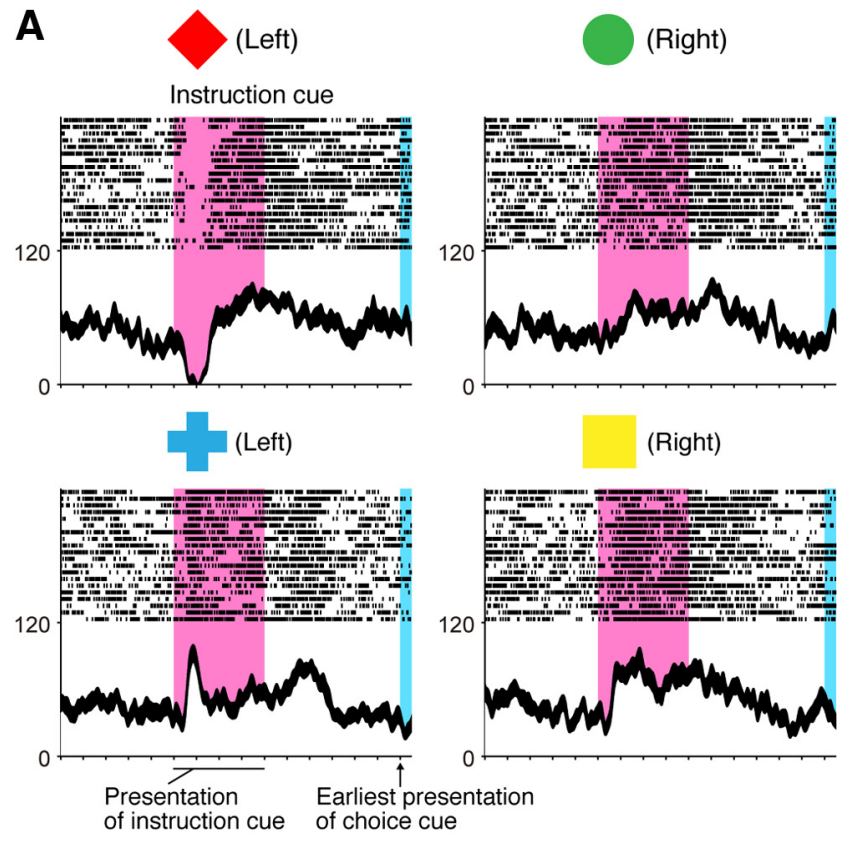

B

(Left)

(Right)

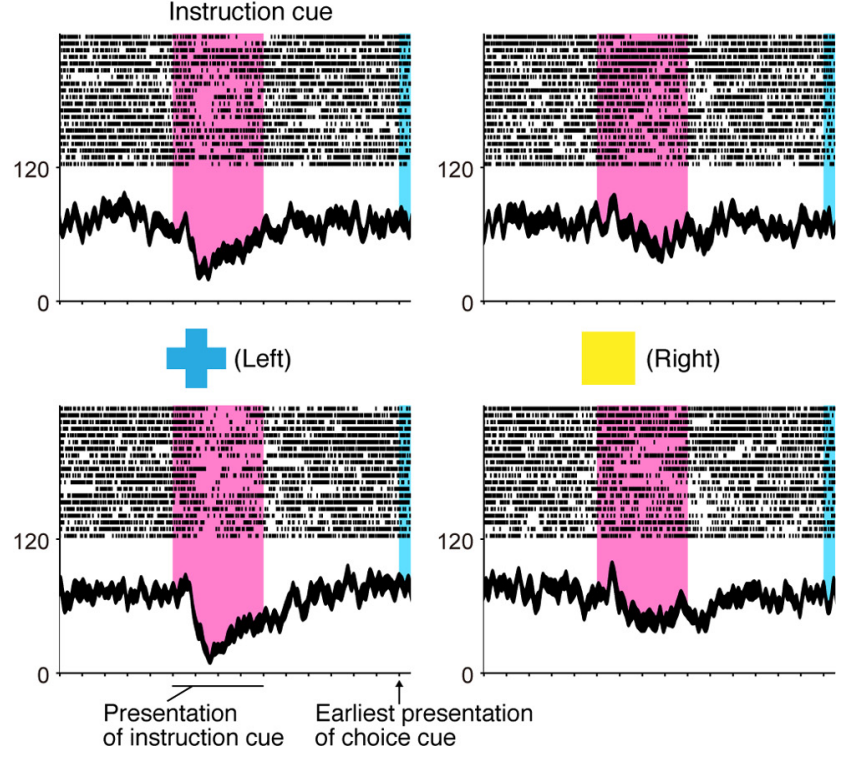

Figure 2. Representative neurons selective for a visual object $(\boldsymbol{A})$ and behavioral goal $(\boldsymbol{B})$ in the symbolic cue task. $A$, Activity of this GP neuron decreased when a red diamond was presented, whereas it increased when a blue cross was presented as an instruction cue. $\boldsymbol{B}$, Activity of this GP neuron decreased when either a red diamond or a blue cross was presented. $\boldsymbol{A}, \boldsymbol{B}$, Neuronal activity was sorted according to the visual object identity. Rasters and spike density functions (smoothed using a Gaussian kernel; $\sigma=10 \mathrm{~ms}$, mean \pm SEM) indicate activity in sorted trials. The ordinate represents the instantaneous firing rate (in spikes/s). Neuronal activity was aligned with the onset of the instruction cue. The pink areas on the left indicate when the instruction was presented, and the blue areas on the right represent the earliest presentation period of the choice cue. The tick marks on the horizontal axis are placed at $200 \mathrm{~ms}$ intervals.

following behavioral factors: instruction-cue object, behavioral goal, choice-cue location, both the behavioral goal and choicecue location, and action. The time course of the appearance of neurons exhibiting each type of selectivity is presented in the histogram shown in Figure $4 A$ for the total GP neurons. After the instruction-cue presentation, 30 neurons (3\%) were classified as selective for visual objects (Fig. 4A; green bars), and 67 neurons (7\%) were classified as selective for behavioral goals (blue bars) in 
Left

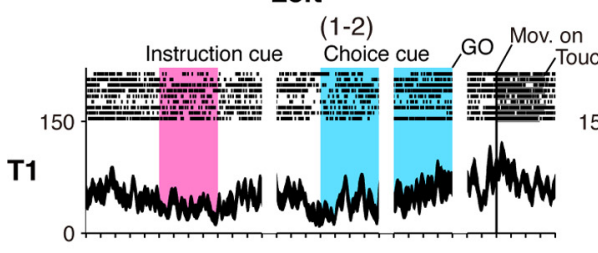

(2-3)

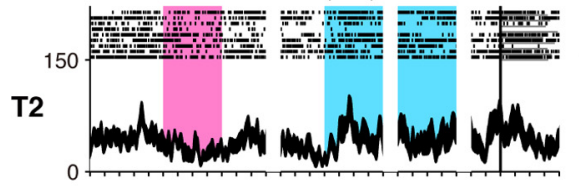

(3-4)

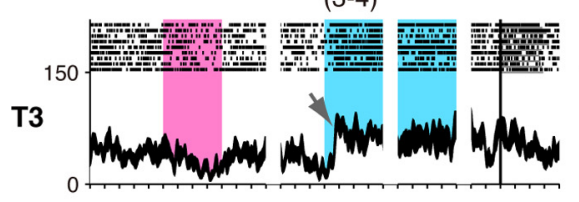

(4-5)

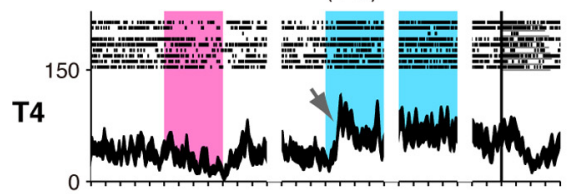

(5-6)

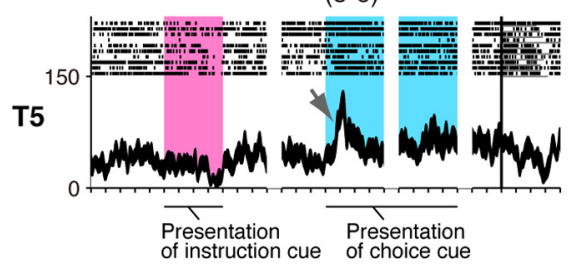

Figure 3. Neuronal activity reflecting action in the symbolic cue task. In this display, neuronal activity is sorted according to the location of the target on the screen (Fig. 1C, T1-T5 rows) as well as to the instructions signaling a left or right future target (left and right columns). This neuron exhibited progressively greater activity after choice-cue presentation as the reach target was placed in positions farther to the right (marked with arrows). Neuronal activity was aligned to the onset of the instruction, choice cue, GO signal, and movement. The pink areas on the left indicate when the instruction was presented, and the blue areas on the right represent the choice-cue presentation period. The location of the choice cue is shown in parentheses (Fig. 1C).
Right

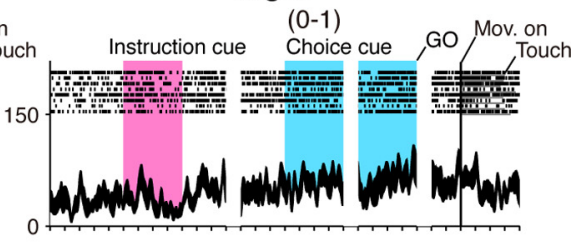

$(1-2)$

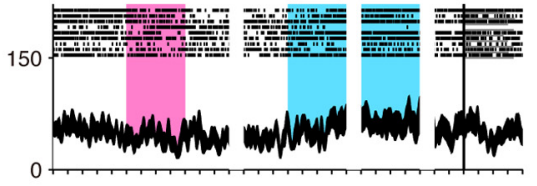

(2-3)

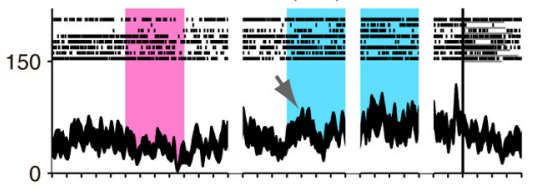

(3-4)

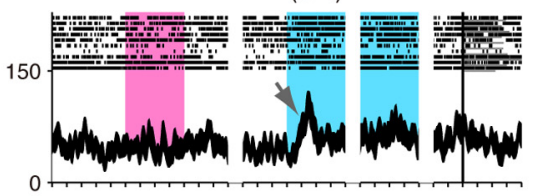

(4-5)

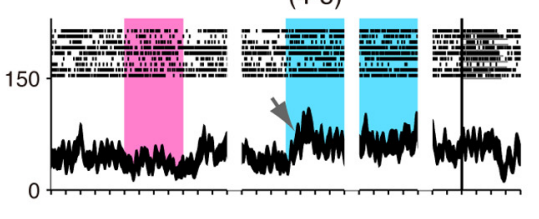

instruction-cue onset (Nakayama et al., 2008; Yamagata et al., 2012). Using the data in these studies and the data in the current study, we compared the proportion of neurons in the GP and cortical areas (i.e., PMd, dlPFC, and vlPFC) that were selective for goals and visual objects. Of the 97 neurons selective for goals ( $n=$ 67 ) or objects $(n=30)$ in the GP, $31 \%$ reflected object features (Fig. 5A; Table 1). The object neurons were observed more frequently in the GP than in the PMd or dlPFC (Fisher's exact test, $p<0.05$; Table $1)$, whereas they were less frequent in the GP than in the vlPFC $(p<0.01)$. These results indicate that the extent of cueobject representation in the GP was intermediate between the values obtained in the vlPFC and in the PMd or dlPFC.

We subsequently examined the magnitude and development of selectivity for the visual objects and goals. For this purpose, we first performed the same ANOVA as above, comparing activity after calculating inverse interspike intervals at a resolution of $10 \mathrm{~ms}$ (Hoshi and Tanji, 2006). We then assessed the initial time point at which neurons began to exhibit selectivity for object features or goals for at least three consecutive $10 \mathrm{~ms}$ bins. The initial time point was defined as the onset of selectivity. For neurons with an onset of selectivity of $\leq 300 \mathrm{~ms}$, we investigated population selectivity separately for excited and inhibited responses. To do that, we first defined an object or an instructed goal that led to the greatest changes in activity (during the $300 \mathrm{~ms}$ period after cue onset) from the control period (during the $500 \mathrm{~ms}$ period preceding cue onset) as the preferred object or goal. When the activity was greater (smaller) than that dur-

the first $200 \mathrm{~ms}$ bin after the instruction-cue onset (i.e., 101-300 $\mathrm{ms}$ after instruction-cue presentation). Figure $4 B, C$ shows the same set of data separately for the GPe (Fig. $4 B$ ) and GPi (Fig. $4 C$ ). The distribution of either object or goal neurons was not different between the GPe and GPi during the period of 101-300 $\mathrm{ms}$ after the instruction-cue onset (Binomial test, $p>0.5$ ).

To investigate the possibility that the neurons classified as goal selective merely responded to two of the four objects, we examined the two objects that led to the highest and second highest activity. This analysis revealed that the combination of a red diamond and a blue cross or of a green circle and a yellow rectangle elicited the highest and second highest activity, respectively, in $69 \%(n=66)$ of the GP neurons that differentially responded to the four instruction cues ( $n=88$; one-way ANOVA, $p<0.01)$. This value exceeded the chance occurrence rate (33\%; binomial test, $p<0.0001$ ). Because both of these object combinations were associated with a particular behavioral goal (left or right), the result suggests that cue-selective neuronal responses in the GP are generally biased toward a behavioral goal.

Our previous studies demonstrated that neurons in the PMd, dlPFC, and vlPFC also represent goals and visual objects after ing the control period, the neuron was considered to exhibit excited (inhibited) responses. For the excited (inhibited) neurons, the nonpreferred object or goal was defined as the one that led to the lowest (highest) discharge rate. We then computed the activity difference between the preferred and nonpreferred object or goal for each $10 \mathrm{~ms}$ and plotted the results separately for the excited and inhibited responses.

Population selectivity showed that both the excited and inhibited responses of GP neurons represent both the visual objects and the goals. We examined the time course of selectivity development by defining it as the point at which the activity modulation exceeded the mean $\pm 4 \mathrm{SD}$ of the baseline period. The responses representing the objects began at $110 \mathrm{~ms}$ for excited neurons $(n=10$; Fig. $6 A)$ and at $170 \mathrm{~ms}$ for inhibited neurons $(n=7$; Fig. $6 A$ ). The absolute magnitude of object selectivity for excited neurons reached 40 spikes/s at $170 \mathrm{~ms}$ after the instruction-cue onset, whereas that for inhibited neurons reached 47 spikes/s at $220 \mathrm{~ms}$. The responses representing the goals began at $140 \mathrm{~ms}$ for excited neurons $(n=26$; Fig. $6 B)$ and at $160 \mathrm{~ms}$ for inhibited neurons $(n=31$; Fig. $6 B)$. The absolute magnitude of goal selectivity for excited neurons reached the 
peak of 16 spikes/s at $230 \mathrm{~ms}$ after the instruction-cue onset, whereas that for inhibited neurons reached 21 spikes/s at 350 ms. These results revealed that both excited and inhibited responses of GP neurons promptly began to represent the object features and the goals after the instruction-cue appearance.

Using the measure of the onset of selectivity as defined above, we compared the development of object and goal representations in the GP with that in the cortical areas (Fig. 7A). As for the object selectivity, the median was $150 \mathrm{~ms}$ in the GP and $200 \mathrm{~ms}$ in the vlPFC (Table 2). The timing of the development in the GP was not significantly different from that in the vlPFC (Kolmogorov-Smirnov test, $p>$ $0.05)$. We subsequently compared the development of goal representation in the GP and the PMd, vlPFC, and dlPFC. The median was $200 \mathrm{~ms}$ in the GP, $180 \mathrm{~ms}$ in the vlPFC, $210 \mathrm{~ms}$ in the dlPFC, and $190 \mathrm{~ms}$ in the PMd (Table 2). The timing was not significantly different between the GP and each of the three cortical areas (KolmogorovSmirnov test, $p>0.05$, Fig. $7 B$ ). These results revealed that the GP began to represent visual objects and behavioral goals no later than the vlPFC, dlPFC, or PMd.

\section{GP neuron activity after}

\section{choice-cue presentation}

GP neurons continued to be selective for behavioral goals after the choice cue was presented. The number of goal-selective neurons during the post-choice-cue period $(n=69,101-300 \mathrm{~ms}$ after the cue onset) was no smaller than the number in the precue period ( $n=70,-299$ to -100 ms preceding the cue). We further studied whether the goal-selective neurons observed in the prechoice-cue period and postchoice-cue period belonged to the same population. We found that, among the 97 neurons selective for the goal around the choice-cue onset $(-99$ to $+100 \mathrm{~ms}$ relative to the onset), only 23 (24\%) neurons continued to be goal selective after the choice-cue onset (101-300 ms relative to the onset; Fig. 8; Table 3 ). These data indicate that a distinct population of neurons began to encode the goals after the choice-cue onset. This result is in accord with findings obtained in the PMd, vlPFC, and dlPFC (Fig. 8; Table 3); in the same comparison, $22 \%$ of PMd neurons ( $n=44$ of 203$), 0 \%$ of vlPFC neurons $(n=$ 0 of 34 ), and $29 \%$ of dlPFC neurons ( $n=35$ of 119) continued to be goal selective after the choice cue onset.

In addition to the goal-selective neurons, three types of neurons were newly
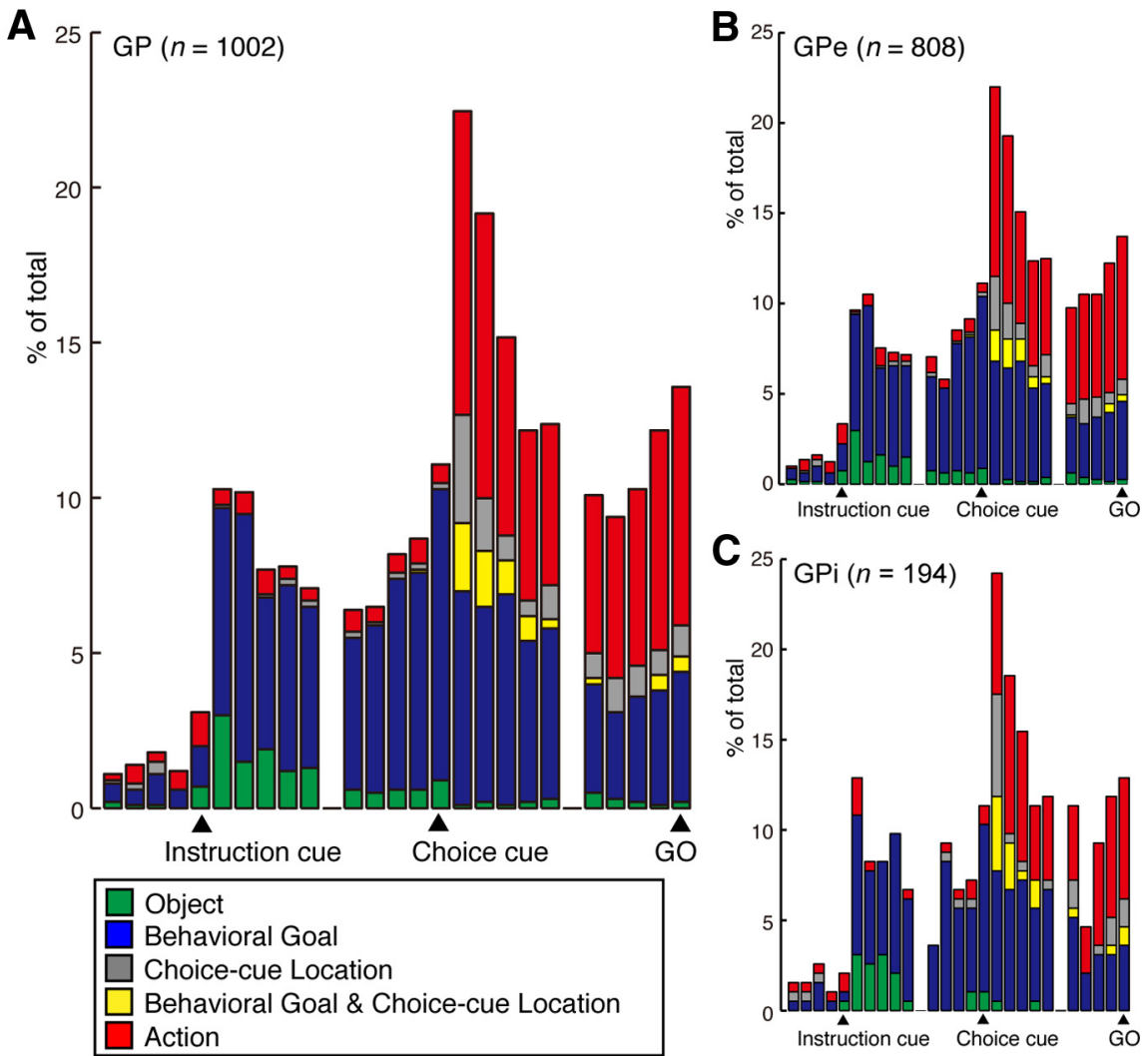

Figure 4. Temporal profiles of the number of selective GP neurons in the symbolic cue task. $\boldsymbol{A}$, The histogram shows the time course of the appearance of neurons exhibiting each type of selectivity for the total GP neurons. $B, C$, Histograms show the same set of data as in $\boldsymbol{A}$ separately for the GPe (B) and GPi (C). The bin width is set to $200 \mathrm{~ms}$. Each category is color-coded: the object (green), behavioral goal (blue), choice-cue location (gray), mixed selectivity for the behavioral goal and choice-cue location (yellow), and action (red). Bins are centered at the instruction-cue onset, choice-cue onset, and G0 onset.
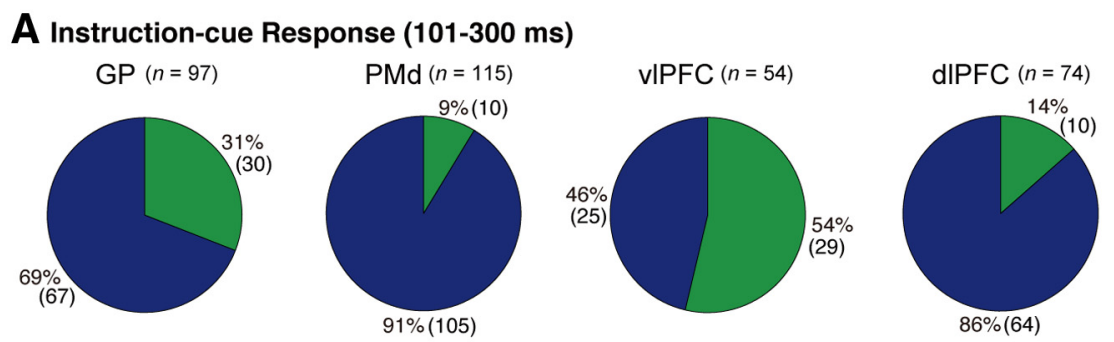

\section{B Choice-cue Response (101-300 ms)}
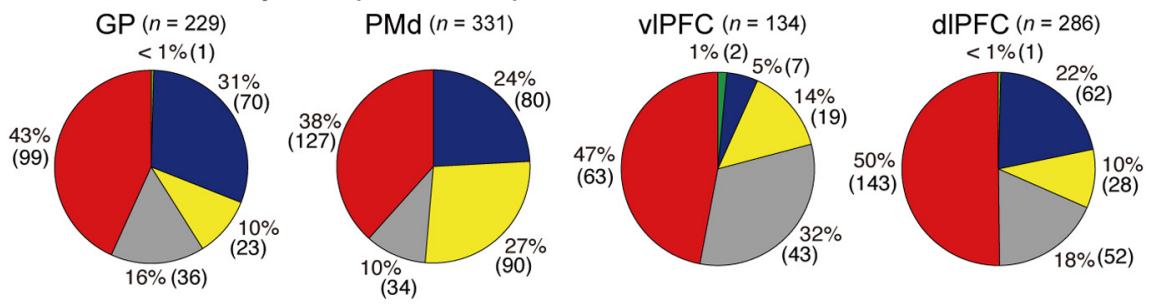

Figure 5. Distribution of selective neurons in the GP, PMd, vIPFC, and dIPFC. Pie charts summarize the proportion of neurons classified into five categories using an ANOVA. Two sets of data are shown during 101-300 ms after the onset of instruction $(\boldsymbol{A})$ and during 101-300 ms after the onset of choice cue $(\boldsymbol{B})$. Each category is color-coded, which is indicated in the inset. Green, object neurons; blue, goal neurons; gray, neurons selective for the choice-cue location; yellow, neurons selective for both goal and choice-cue location; red, action neurons. 
Table 1. Fraction of selective neurons in the GP, PMd, vIPFC, and dIPFC after instruction-cue onset $^{a}$

\begin{tabular}{lcccl}
\hline & \multicolumn{4}{l}{$101-300$ ms relative to instruction-cue onset } \\
\cline { 2 - 5 } Area & Object & Behavioral goal & Total of selective & Nonselective \\
\hline $\mathrm{GP}(n=1002)$ & $30(31)$ & $67(69)$ & 97 & 899 \\
PMd (Monkey 1, $n=330)$ & $10^{* *}(9)$ & $105^{* *}(91)$ & 115 & 215 \\
$\operatorname{vIPFC}(n=358)$ & $29^{* *}(54)$ & $25^{* *}(46)$ & 54 & 301 \\
$\operatorname{dIPFC}(n=791)$ & $10^{*}(14)$ & $64^{*}(86)$ & 74 & 709
\end{tabular}

${ }^{a}$ Data indicate the number of neurons exhibiting selectivity to the objects and to the behavioral goals $101-300 \mathrm{~ms}$ after the instruction-cue onset; values in parentheses indicate the percentage of neurons out of the total of the object- and goal-neurons.

${ }^{*} p<0.05,{ }^{* *} p<0.01$ between the GP and each cortical area (Fisher's exact test).

\section{A Symbolic-cue task, Object}

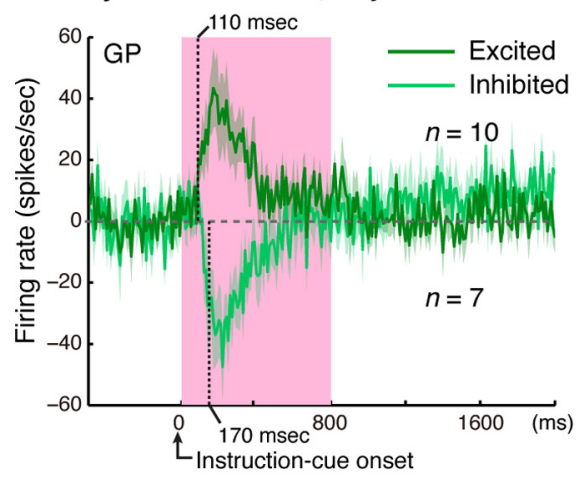

B Symbolic-cue task, Behavioral goal

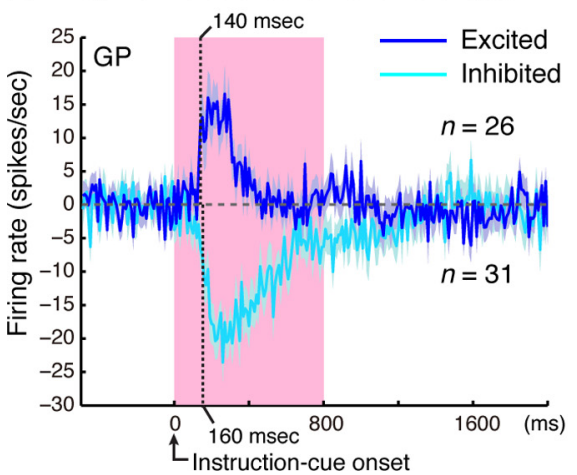

\section{Symbolic-cue task, Action}

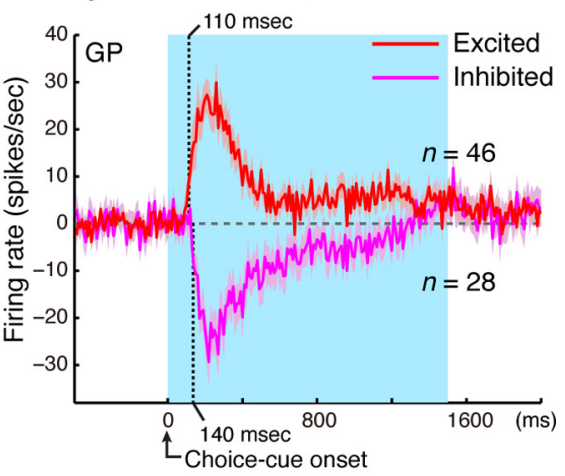

\section{Direct-cue task, Visuospace}

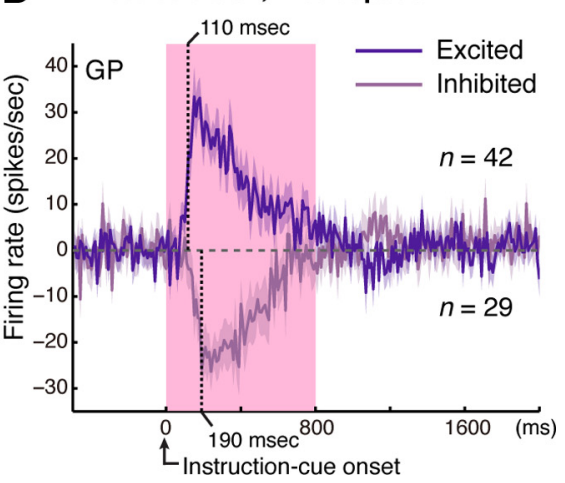

Figure 6. Development of selective responses. Time courses of object selectivity $(\boldsymbol{A})$, behavioral goal selectivity $(\boldsymbol{B})$, action selectivity $(\boldsymbol{C})$ in the symbolic cue task, and visuospatial selectivity in the direct-cue task $(\boldsymbol{D})$. Bin-by-bin plots of the mean activity modification ( \pm SEM) were calculated as the difference in activity for the preferred and nonpreferred cases. Analyses were performed for neurons exhibiting selective responses after presentation of the instruction cue $(\boldsymbol{A}, \boldsymbol{B}, \boldsymbol{D})$ or choice cue $(\boldsymbol{C})$. Dark green $(\boldsymbol{A})$, blue $(\boldsymbol{B})$, red $(\boldsymbol{C})$, and purple $(\boldsymbol{D})$ lines represent excited responses; and light green $(\boldsymbol{A})$, light blue $(\boldsymbol{B})$, pink $(\boldsymbol{C})$, and light purple (D) lines represent inhibited responses. $\boldsymbol{A}, \boldsymbol{B}, \boldsymbol{D}$, Pink areas indicate when the instruction cues were presented. $\boldsymbol{C}$, Blue areas indicate the minimum period of choice-cue presentation. Translucent ribbons around the solid line indicate $\pm S E M$. Each vertical dotted line represents the onset latency: a point at which the selectivity exceeds the mean \pm 4 SD of the baseline period (500 ms period preceding the cue onset).

observed during the postcue period: (1) neurons selective for the choice-cue location, (2) neurons selective for both the behavioral goal and the choice-cue location (mixed selectivity), and (3) neurons selective only for the action (Fig. $4 A$ for the total GP neurons). During the 101-300 ms after the choice-cue onset (Fig. $5 B), 99$ neurons were selective for the action, whereas 36 neurons were selective for the choice-cue location, and 23 neurons for both the behavioral goal and the choice-cue location (Table 4). Figure $4 B, C$ shows the same set of data separately for the GPe (Fig. $4 B$ ) and GPi (Fig. 4C). The distribution of neurons with each type of selectivity was not different between the GPe and GPi
(Binomial test, $p>0.05$ ) during the period of $101-300 \mathrm{~ms}$ after we onset. distribution observed in the frontal cortex by comparing the ocnces of task-selective neurons during the early part of the (t) GP was comparable with those in the dIPFC (Fisher's exact test, $p \geq 0.1$; Fig. $5 B$ ). In contrast, the distribution among GP neurons differed from the distribution reported in the vlPFC and the PMd. Neurons selective for the choicecue location were found more frequently in the vlPFC than in the GP (Fisher's exact test, $p<0.01$; Table 4 ), and neurons with mixed selectivity for both the goal and the choice-cue location were more frequent in the PMd than in the GP $(p<0.01$; Table 4). Furthermore, out of neurons selective for the goal around the choice cue onset (Fig. 8, column A, blue), fewer neurons in the GP than in the PMd exhibited selectivity for a mixture of the goal and the choice-cue location after the choice-cue onset (Fig. 8, column B, yellow; Table 3; Fisher's exact test, $p<$ 0.01 ), suggesting that goal representation in the GP is only rarely used for the direct interaction between the goal and the choice-cue location.

For the action-selective neurons in the GP, we subsequently investigated the time course of the postcue development of selectivity among the population of neurons belonging to that category. After performing the same ANOVA as above on activity after calculating inverse interspike intervals with a resolution of $10 \mathrm{~ms}$, we examined an initial time point (i.e., the onset of action selectivity) at which a neuron began to exhibit action selectivity for at least three consecutive $10 \mathrm{~ms}$ bins after the choice-cue onset. For neurons with a selectivity onset of $\leq 300 \mathrm{~ms}$, we investigated population selectivity separately for excited and inhibited responses. We defined an action (i.e., the target position on the screen) that led to the greatest changes in activity (during the $300 \mathrm{~ms}$ period after the choice-cue onset) from the control period (500 ms period preceding the choicecue onset) as the preferred action. When the activity was greater (smaller) than that during the control period, the neuron was considered to exhibit excited (inhibited) responses. For the excited (inhibited) neurons, the nonpreferred action was defined as the action with the lowest (highest) discharge rate. We then calculated the difference in activity between the preferred and nonpreferred actions for each $10 \mathrm{~ms}$ and plotted the results separately for the excited and inhibited responses (Fig. 6C).

We subsequently examined the latency of the development of action selectivity by defining it as the point at which the activity modulation exceeded the mean $\pm 4 \mathrm{SD}$ of the baseline period. 


\section{A Symbolic cue task, Object}

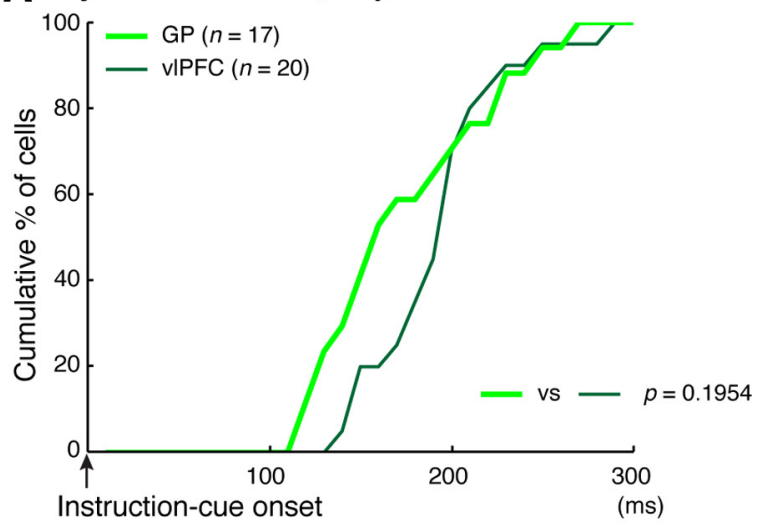

B Symbolic cue task, Behavioral goal

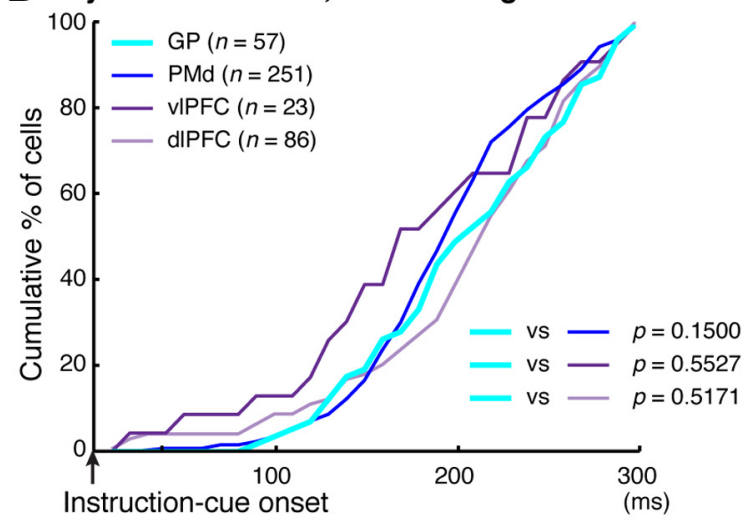

C Symbolic cue task, Action

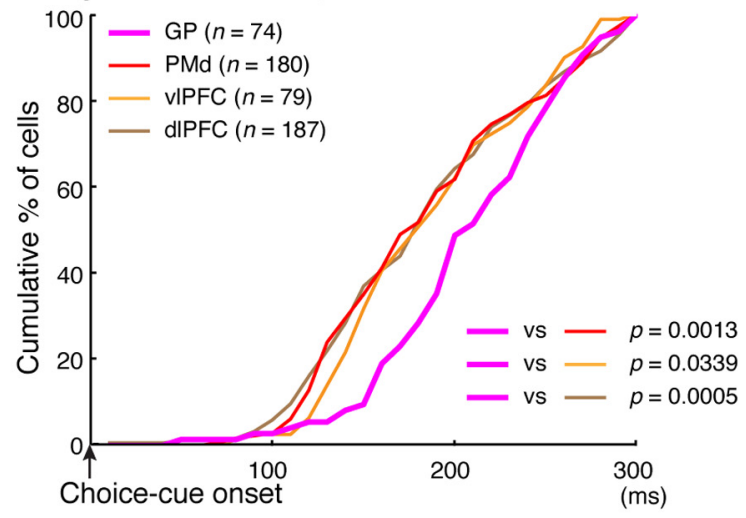

D Direct-cue task, Visuospace

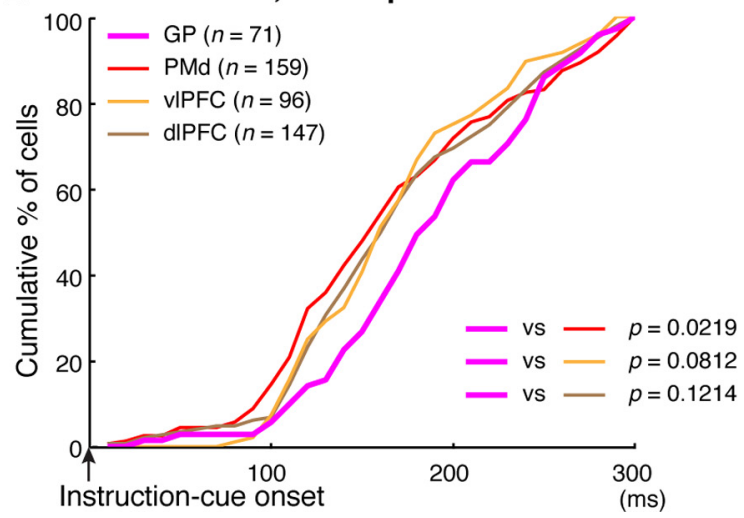

Figure 7. Comparison of activity-selective development for the visual objects, behavioral goals, and actions. $A$, Cumulative fractions of selectivity onset for visual objects after the instruction
Table 2. Median onset latency of selective activity after instruction-cue and choicecue onset $^{a}$

\begin{tabular}{lllll}
\hline & \multicolumn{3}{l}{ After instruction-cue onset } & \\
\cline { 2 - 4 } Area & Object & Behavioral goal & Visuospace (direct-cue task) & After choice-cue onset: action \\
\hline GP & 150 & 200 & 180 & 200 \\
PMd & N/A & 190 & 150 & 170 \\
vIPFC & 200 & 180 & 150 & 170 \\
dIPFC & N/A & 210 & 150 & 170 \\
\hline
\end{tabular}

Data are in milliseconds.

The responses representing the action began at $110 \mathrm{~ms}$ for excited neurons ( $n=46$; Fig. $6 C$ ) and at $140 \mathrm{~ms}$ for inhibited neurons $(n=28)$. The absolute magnitude of action selectivity for excited neurons reached the peak of 28 spikes/s at $260 \mathrm{~ms}$ after the choice-cue onset, whereas that for excited neurons reached the peak of 27 spikes/s at $220 \mathrm{~ms}$. These results revealed that both excited and inhibited responses of GP neurons promptly began to represent the actions after the choice cue appearance.

Using the measure of the onset of action selectivity described above, we compared the development of action selectivity in the GP with that in the vlPFC, dlPFC, and PMd. Figure $7 C$ shows a cumulative distribution of the onset of action selectivity among neurons in these areas. The median was $200 \mathrm{~ms}$ in the GP, whereas it was $170 \mathrm{~ms}$ in the vlPFC, dlPFC, and PMd (Table 2). Action representation in the GP developed later than it did in the vlPFC, dlPFC, and PMd (Kolmogorov-Smirnov test, $p<0.05$; Fig. $7 C)$. These results revealed that action representation initially developed in the cortical areas, followed by the GP.

\section{Visuospatial responses of GP neurons}

The above observation that action selectivity developed later in the GP than in the cortical areas raised the intriguing possibility that the visuospatial information itself may reach the GP later than it reaches the cortical areas. To investigate this, we examined the visuospatial responses in the direct cue task (Fig. 1D). In this task, a visual signal was presented in one of five horizontal arrays (Fig. $1 E$ ) while the monkeys gazed at a fixation point. After calculating inverse interspike intervals with a resolution of $10 \mathrm{~ms}$, we performed a one-way ANOVA with the five positions as independent variables. We then identified an initial time point at which a neuron began to exhibit selectivity for the spatial position for at least three consecutive $10 \mathrm{~ms}$ bins and defined this as the onset of visuospatial selectivity. For neurons with a selectivity onset of $\leq 300 \mathrm{~ms}$, we computed the population selectivity (Fig. $6 D$ ) in the same manner as for the action selectivity (Fig. $6 \mathrm{C}$ ); the only difference was that the instruction-cue position in the direct cue task (Fig. 1E, T1-T5) was used instead of the action in the symbolic cue task (Fig. 1C, T1-T5). The results show that excited responses in the GP began $110 \mathrm{~ms}$ after the cue onset $(n=42$, Fig. $6 D)$, whereas inhibited responses in the GP began $190 \mathrm{~ms}$ after the onset $(n=29)$. The absolute magnitude of visuospatial selectivity

$\leftarrow$

cue onset in the GP (light green) and vIPFC (dark green) in the symbolic cue task. B, Cumulative fractions of selectivity onset for the behavioral goal after instruction-cue presentation in the GP (light blue), PMd (dark blue), vIPFC (dark purple), and dIPFC (light purple) in the symbolic cue task. C, Cumulative fractions of action-selectivity onset after the choice-cue onset in the GP (pink), PMd (red), vIPFC (orange), and dIPFC (brown) in the symbolic cue task. D, Cumulative fractions of selectivity onset for cue visuospatial position in the direct-cue task after the instruction-cue onset in the GP (pink), PMd (red), vIPFC (orange), and dIPFC (brown). $\boldsymbol{A}-\boldsymbol{D}, \boldsymbol{p}$ values indicate the results of the statistical analysis (Kolmogorov-Smirnov test) between the $\mathrm{GP}$ and the other three areas. 


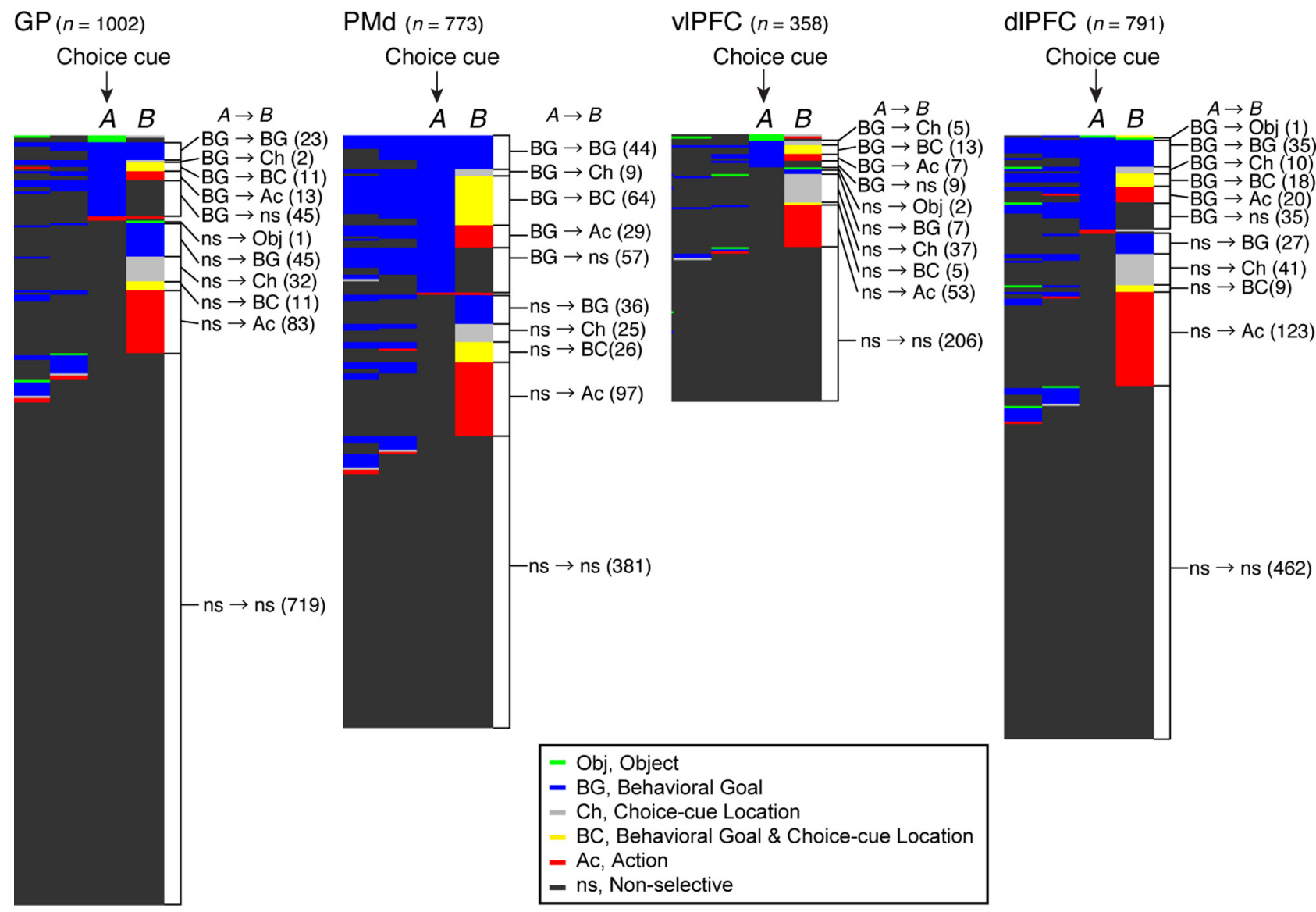

Figure 8. Time-dependent selectivity of each neuron for each behavioral factor. For each $200 \mathrm{~ms}$ time bin in the task period, individual neuron properties were classified into one of six categories based on their specific selectivity for each of the behavioral factors. Activity profiles of individual neurons are displayed consecutively. Each row represents data from a single neuron. Data from the GP, PMd, vIPFC, and dIPFC are displayed separately. In each panel, neurons were sorted according to selectivity at the choice-cue onset (A). To the right of each panel, we compared the selectivity of each neuron in two distinct bins (at A and B). Different colors indicate the selectivity for the object (Obj, green), behavioral goal (BG, blue), choice-cue location (Ch, gray), combined behavioral goal and choice-cue location (BC, yellow), action ( $A c$, red), and the nonselective neuron (ns, black). The numbers in parentheses indicate the number of neurons in each group.

for excited neurons reached the peak of 24 spikes/s at $170 \mathrm{~ms}$ after the instruction-cue onset, whereas that for inhibited neurons reached the peak of 22 spikes/s at $230 \mathrm{~ms}$. These results revealed that the visuospatial information was promptly encoded by both excited and inhibited responses of GP neurons after the cue appearance.

Finally, using the measure of the onset of visuospatial selectivity, we compared the development of visuospatial representation in the GP with that in the vlPFC, dlPFC, and PMd (Fig. 7D). The median was $180 \mathrm{~ms}$ in the GP, whereas it was $150 \mathrm{~ms}$ in the vlPFC, dlPFC, and PMd (Table 2). Thus, the development of visuospatial selectivity in the GP tended to follow that in the cortical areas. The statistical analysis revealed that development in the GP was significantly later than that in the PMd (Kolmogorov-Smirnov test, $p<0.05$; Fig. 7D).

\section{Spatial distribution of selective activity}

Figure 9 shows the distributions of the neurons selective for the visual object, behavioral goal, and action separately for the GPe and GPi; the selectivity was identified via ANOVA applied to the activity of the $200 \mathrm{~ms}$ bins (Fig. 4). After the onset of the instruction and choice cues, neurons coding the behavioral goal were found primarily at the center of the rostrocaudal and mediolateral axes of the GPe and GPi (Fig. 9A,B). Although the distribution of object neurons largely corresponded to that of goal neurons, a subset of object neurons was observed at the caudal part the GPi (Fig. 9C). Similar to goal neurons, neurons selective for action during the choice-cue period were localized in the central portion of the GPe and GPi (Fig. 9D).

\section{Duration of neuronal representations for behavioral goal and action}

From a theoretical perspective (Houk and Wise, 1995), it was of interest to know how long the selectivity of GP neurons for key behavioral elements was held during successive stages in the behavioral task. We compared the duration of goal and action representations among GP neurons with that among PMd, dlPFC, and vlPFC neurons. After performing the ANOVA at a resolution of $10 \mathrm{~ms}$, we then assessed the initial time point at which neurons began to exhibit selectivity for goal or action at least three consecutive $10 \mathrm{~ms}$ bins (the onset of selectivity) and the first time point at which neurons ceased to exhibit selectivity for goal or action at least three consecutive $10 \mathrm{~ms}$ bins (the offset of selectivity).

The distributions of goal selectivity in time after the instruction-cue onset are shown for neurons in the GP (Fig. 10A), PMd (Fig. 10B), vlPFC (Fig. 10C), and dlPFC (Fig. 10D); the blue dots indicate the time points at which each neuron was selective for the goals. We determined the duration of goal selectivity for each neuron by finding the onset and the offset of goal selectivity. The cumulative distributions of goal selectivity summarized for each area (Fig. 10E) revealed that the duration of the GP neurons 
Table 3. Behavioral selectivity relative to choice-cue onset ${ }^{a}$

\begin{tabular}{|c|c|c|c|}
\hline \multirow[b]{2}{*}{$\begin{array}{l}\text { Selectivity } 101-300 \text { ms relative to } \\
\text { choice-cue onset (B) }\end{array}$} & \multicolumn{3}{|c|}{$\begin{array}{l}\text { Selectivity }-99 \text { to } 100 \text { ms relative } \\
\text { to choice-cue onset }(A)\end{array}$} \\
\hline & $\begin{array}{l}\text { Behavioral } \\
\text { goal }\end{array}$ & $\begin{array}{l}\text { Nonbehavioral } \\
\text { goal }\end{array}$ & Nonselective \\
\hline \multicolumn{4}{|l|}{ GP } \\
\hline Object & $0(0)$ & $0(0)$ & $1(0)$ \\
\hline Behavioral goal & $23(2)$ & $2(0)$ & $45(4)$ \\
\hline Choice-cue location & $2(0)$ & $2(0)$ & $32(3)$ \\
\hline Mixed & $11(1)$ & $1(0)$ & $11(1)$ \\
\hline Action & $13(1)$ & $3(0)$ & $83(8)$ \\
\hline Nonselective & $45(4)$ & $9(1)$ & $719(72)$ \\
\hline Total & $94(9)$ & $17(2)$ & $891(89)$ \\
\hline \multicolumn{4}{|l|}{ PMd } \\
\hline Behavioral goal & $44^{* *}(6)$ & $0(0)$ & $36(5)$ \\
\hline Choice-cue location & $9^{*}(1)$ & $0(0)$ & $25(3)$ \\
\hline Mixed & $64^{* *}(8)$ & $0(0)$ & $26^{* *}(3)$ \\
\hline Action & $29 * *(4)$ & $1(0)$ & $97^{* *}(13)$ \\
\hline Nonselective & $57^{*}(7)$ & $4(1)$ & $381^{* *}(49)$ \\
\hline Total & $203(26)$ & $5(1)$ & $565(73)$ \\
\hline \multicolumn{4}{|l|}{ VIPFC } \\
\hline Object & $0(0)$ & $0(0)$ & $2(1)$ \\
\hline Behavioral goal & $0^{* *}(0)$ & $0(0)$ & $7^{*}(2)$ \\
\hline Choice-cue location & $5^{*}(1)$ & $1(0)$ & $37^{* *}(10)$ \\
\hline Mixed & $13^{* *}(4)$ & $1(0)$ & $5(1)$ \\
\hline Action & $7(2)$ & $3(1)$ & $53^{* *}(15)$ \\
\hline Nonselective & $9(3)$ & $9(3)$ & $206^{* *}(58)$ \\
\hline Total & $34(10)$ & $14(4)$ & 310 (87) \\
\hline \multicolumn{4}{|l|}{ dIPFC } \\
\hline Object & $1(0)$ & $0(0)$ & $0(0)$ \\
\hline Behavioral goal & $35^{*}(4)$ & $0(0)$ & $27(3)$ \\
\hline Choice-cue location & $10^{* *}(1)$ & $1(0)$ & $41^{*}(5)$ \\
\hline Mixed & $18(2)$ & $1(0)$ & $9(1)$ \\
\hline Action & $20(3)$ & $0(0)$ & $123^{* *}(16)$ \\
\hline Nonselective & $35(4)$ & $8(1)$ & $462^{* *}(58)$ \\
\hline Total & $119(15)$ & $10(1)$ & 662 (84) \\
\hline
\end{tabular}

${ }^{a}$ Data indicate the number of neurons exhibiting selectivity to the five aspects of behavioral factors -99 to $100 \mathrm{~ms}$ (A) and 101 to $300 \mathrm{~ms}$ (B) relative to the choice-cue onset; A and B correspond to the bins indicated in Figure 8. The values in parentheses indicate the percentage of neurons of the total ( $n=1002$ in the GP, $n=773$ in the PMd, $n=$ 358 in the vIPFC, $n=791$ in the dIPFC).

${ }^{*} p<0.05,{ }^{* *} p<0.01$ between the GP and each of PMd, vIPFC, and dIPFC (Fisher's exact test).

was significantly shorter than that of the PMd and dlPFC neurons (Kolmogorov-Smirnov test, $p<0.0001$ ), whereas the duration was not significantly different between the GP and vlPFC neurons $(p=0.3403)$.

We subsequently examined the duration of action selectivity after the choice-cue onset. The distributions of action selectivity in time are shown for neurons in the GP (Fig. 10F), PMd (Fig. $10 G$ ), vlPFC (Fig. 10H), and dlPFC (Fig. 10I); the red dots indicate the time points at which each neuron was selective for the actions. Based on this analysis, we determined the duration of action selectivity for each neuron. The cumulative distributions of the action durations summarized for each area (Fig. 10J) revealed that the duration of action selectivity of the GP neurons was significantly shorter than that of the PMd, vlPFC, and dlPFC neurons (Kolmogorov-Smirnov test, $p<0.0001$ for PMd, $p=$ 0.0120 for vlPFC, $p=0.0002$ for dlPFC).

These analyses revealed that the durations of the neuronal representations for both the behavioral goal and the action were shorter in the GP than in the PMd and dlPFC, whereas the durations of the neuronal representations only for the action were shorter in the GP than in the vlPFC.

\section{Discussion}

We compared the response of neurons in the dorsal GP with those in the PMd and IPFC while monkeys performed a task requiring the retrieval of abstract behavioral goals and the specification of forthcoming actions to achieve those goals. We found that GP neurons exhibited activity representing task-relevant signals in common with neurons in the PMd and lPFC. In contrast, several specific aspects of GP neuronal activity differed from those of neurons in these cortical areas. Functional implications of these findings are discussed below.

\section{Representation of visual objects and visuospatial signals in the GP}

We found that GP neurons represented features of visual objects. A subset of object-selective neurons was found in the caudal and lateral portion of internal segment of the GP. The inferotemporal cortex projects to the ventral region of the middle to caudal portion of the striatum (Webster et al., 1993; Middleton and Strick, 1994; Cheng et al., 1997), where neurons respond to visual stimuli in an object-selective manner (Caan et al., 1984; Brown et al., 1995; Yamamoto et al., 2012). Because of this topographical organization, this striatal portion appears to project to the caudal part of the GPi, where we observed object neurons (Alexander and Crutcher, 1990; Gimenez-Amaya and Graybiel, 1990; SaintCyr et al., 1990; Flaherty and Graybiel, 1993; Mink and Thach, 1993; Parent and Hazrati, 1995a, b; Kaneda et al., 2002). Another subset of object neurons was found in the dorsal portion of the GP. These neurons may receive inputs from the ventral prefrontal cortex, where object information is well represented (Scalaidhe et al., 1997). By examining the response latency, we found that object feature representation appeared indistinguishably in the GP and vlPFC, although the median value for GP neurons was shorter than that for vlPFC neurons by $50 \mathrm{~ms}$. If we could compare more numerous neurons, we might have found that the latency was shorter in the GP than in the vlPFC. In either case, the prompt response in the GP may originate from the enhanced coupling between the inferotemporal cortex and the striatum that is shown in subjects who have learned visuomotor associations (Toni et al., 2002).

We also found that GP neurons represent visuospatial signals (Kato and Hikosaka, 1995; Shin and Sommer, 2010). The visuospatial neurons responding to the choice cue were centered rostrocaudally on the middle portion of the dorsal GP, which projects across synapses to the premotor cortex and the IPFC (Middleton and Strick, 2002; Saga et al., 2011). Thus, spatial information may originate from the premotor cortex as well as from the posterior parietal cortex (Selemon and Goldman-Rakic, 1985). In the direct-cue task, the visuospatial responses tended to appear later in the GP than in the PMd and IPFC, suggesting that the GP may not play a major role in initiating prompt responses to visuospatial signals in these cortical areas. Furthermore, in the symbolic-cue task, neurons reflecting the choice-cue location were found less frequently in the GP than in the vlPFC. This result, together with our finding that the object-selective GP neurons responding to the instruction cue appeared less frequently than in the vlPFC, indicates that GP neurons are less readily driven by visual signals than are vlPFC neurons. This may be attributable to the fact that the GP receives major inputs from the cerebral cortex indirectly via the subthalamic nucleus and the striatum (Hazrati and Parent, 1992; Ryan and Sanders, 1994; Maurice et al., 1998, 1999; Nambu et al., 2000; Kita et al., 2004; Tachibana et al., 2008).

\section{Development of abstract behavioral goals in the GP}

BG involvement in associating visual signals with actions has been established in both monkeys and humans (see Introduc- 
Table 4. Fraction of selective neurons in the GP, PMd, vIPFC, and dIPFC after choice-cue onset ${ }^{a}$

\begin{tabular}{|c|c|c|c|c|c|c|c|}
\hline \multirow[b]{2}{*}{ Area } & \multicolumn{7}{|c|}{ Selectivity during 101-300 ms after choice-cue onset } \\
\hline & Object & Behavioral goal & Mixed & Choice-cue location & Action & Total of selective & Nonselective \\
\hline $\mathrm{GP}(n=1002)$ & $1(0)$ & $70(31)$ & $23(10)$ & $36(16)$ & $99(43)$ & 229 & 773 \\
\hline $\operatorname{PMd}(n=773)$ & $\mathrm{N} / \mathrm{A}$ & $80(24)$ & $90^{* *}(27)$ & $34(10)$ & $127(38)$ & 331 & 442 \\
\hline $\operatorname{vIPFC}(n=358)$ & $2(1)$ & $7^{* *}(5)$ & 19 (14) & $43^{* *}(32)$ & $63(47)$ & 134 & 224 \\
\hline $\operatorname{dIPFC}(n=791)$ & $1(0)$ & $62^{*}(22)$ & $28(10)$ & $52(18)$ & $143(50)$ & 286 & 505 \\
\hline
\end{tabular}

${ }^{a}$ Data indicate the number of neurons exhibiting selectivity to the five aspects of behavioral factors $101-300 \mathrm{~ms}$ after the cue onset; values in parentheses indicate the percentage of the total neurons.

${ }^{*} p<0.05,{ }^{* *} p<0.01$ between the GP and each area (Fisher's exact test).

\section{A Behavioral goal (Instruction-cue Response) number of cells ○ जे ले \&}

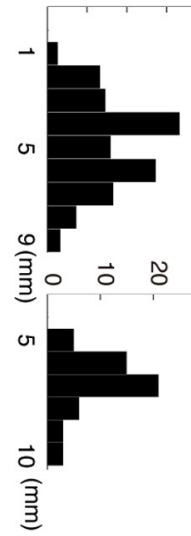

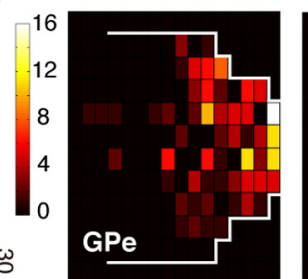

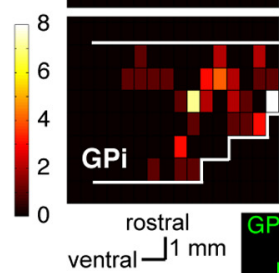

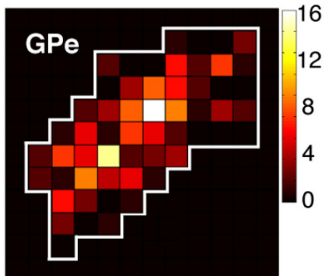

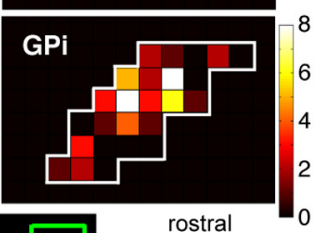
$1 \mathrm{mmL}$ medial

\section{Object (Instruction-cue Response)}

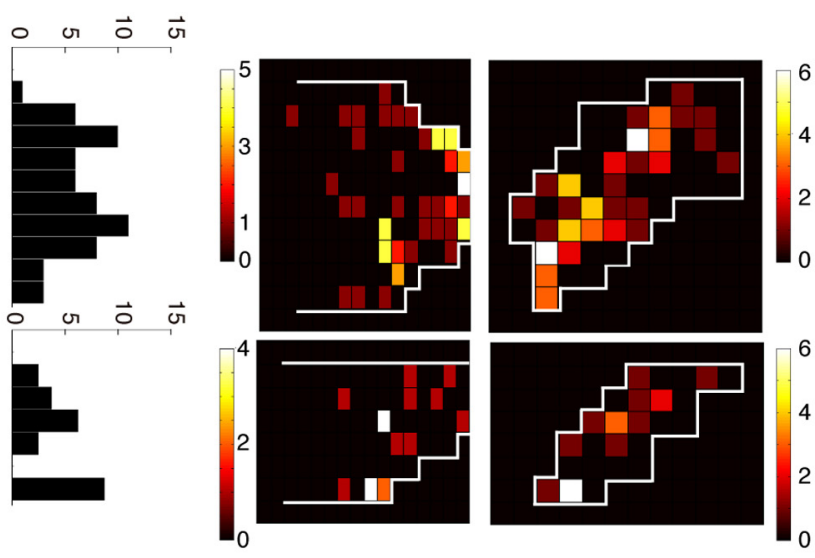

\section{B Behavioral goal (Choice-cue Response)}
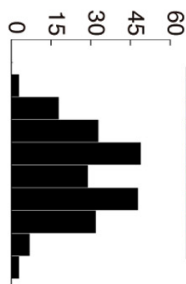

- $r \vec{\circ} \overrightarrow{\mathrm{N}}$

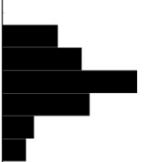

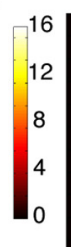

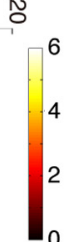

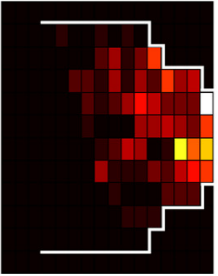

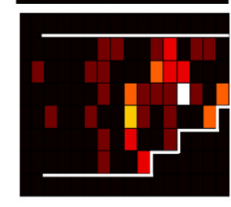

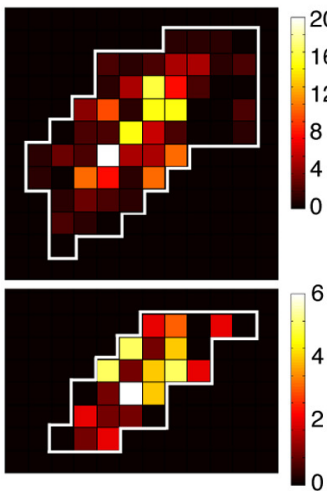
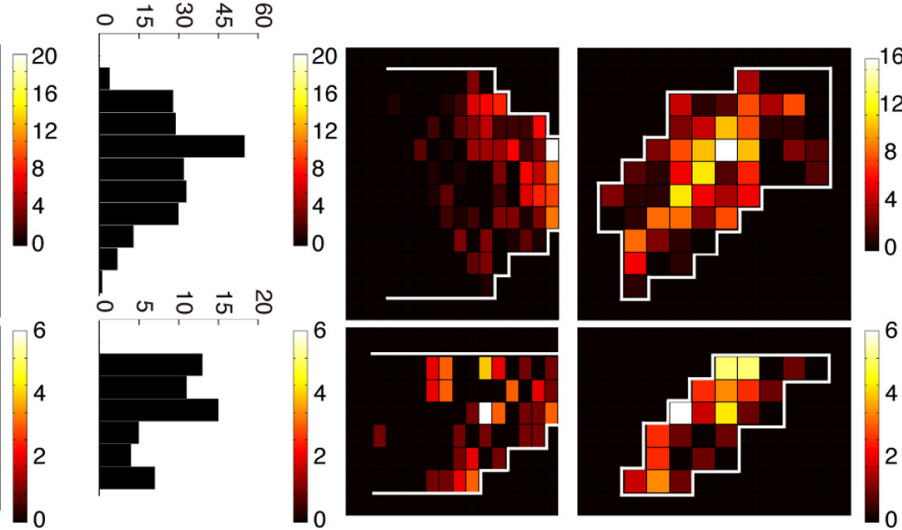

Figure 9. Density distributions of cue-responsive neurons in the GP in the symbolic cue task. Horizontal (right) and sagittal (middle) section maps of the GPe (top) and GPi (bottom) and show the distribution of neurons selective for the behavioral goal $(\boldsymbol{A}, \boldsymbol{B})$, the visual object $(\boldsymbol{C})$, and the action $(\boldsymbol{D})$. The distribution is expressed as the number of neurons with significant selectivity during 101-300, 301-500, 501-700, or 701-900 ms after the cue onset (Fig. 4). The fraction was calculated after each neuron projected into the horizontal (right) and sagittal (middle) planes. Data from two animals are overlaid with reference to the central portion of each GPe and GPi. The color scale indicates the fraction of selective neurons (among all task-related neurons within each miniature portion). Left, Histogram adds the total number of selective neurons within each 1-mm-thick GP slice vertical to the rostrocaudal axis. $\boldsymbol{A}$, bottom right, Line drawings inset indicates approximate spatial locations of GPe (green) and GPi (yellow) along the mediolateral and rostrocaudal axis.

tion). In these studies, however, once an instruction cue was presented, the subjects could determine the actual movements themselves. In contrast, the design of our behavioral task allowed us to dissociate neuronal activity reflecting abstract behavioral goals from that reflecting physical actions. We found that a subset of GP neurons represent behavioral goals in advance of the specification of motor action. Furthermore, behavioral goal development in the GP was comparable with that in vlPFC, dlPFC, and
PMd. These findings indicate that the GP is involved in the initial stages of the computations involved in retrieving behavioral goals from visual instruction signals when associations are established. Clinical studies have reported that BG dysfunction results in deficits in cognitive processes (Mendez et al., 1989; Dubois and Pillon, 1997; Crucian and Okun, 2003; Uc et al., 2005). The loss of neurons representing abstract aspects of behavior may underlie these deficits. 
A Behavioral Goal (GP)

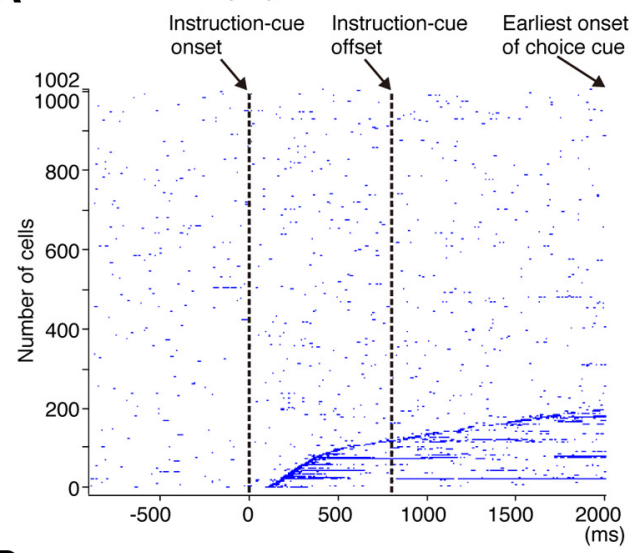

B Behavioral Goal (PMd)

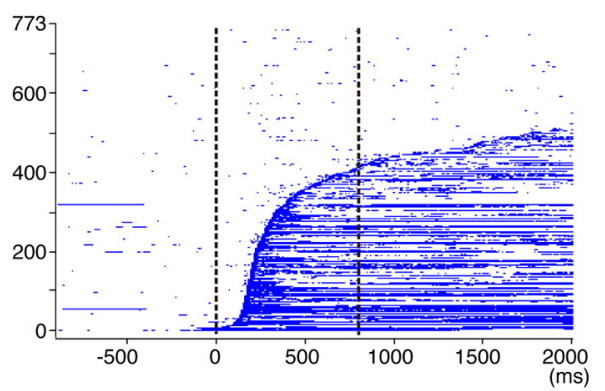

C Behavioral Goal (vIPFC)

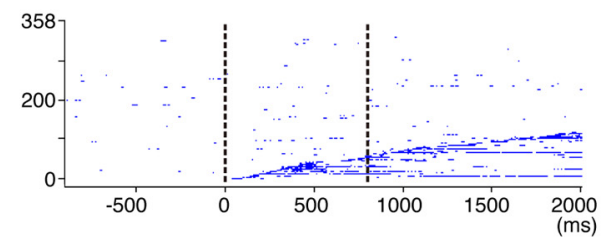

D Behavioral Goal (dIPFC)

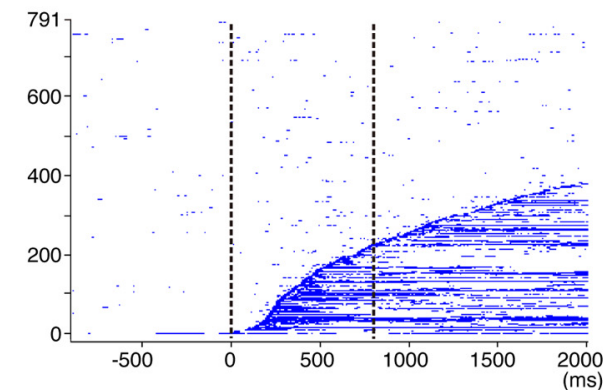

E Duration of selectivity (Behavioral Goal)

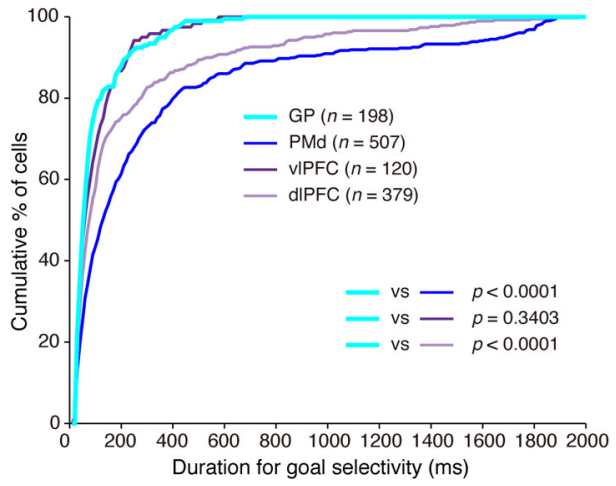

F Action (GP)

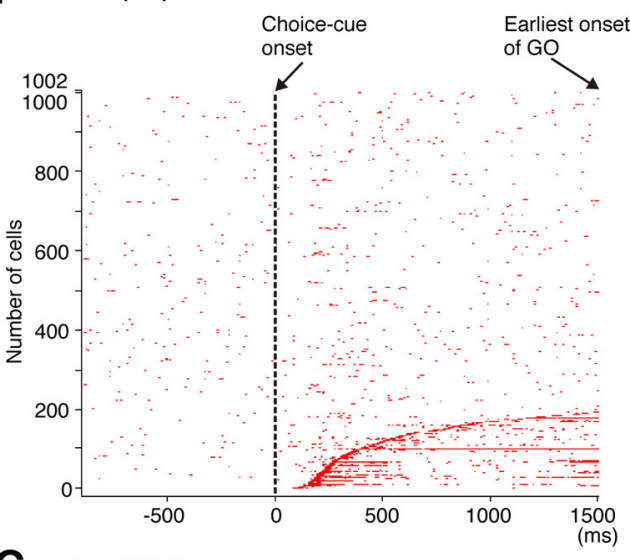

G Action (PMd)

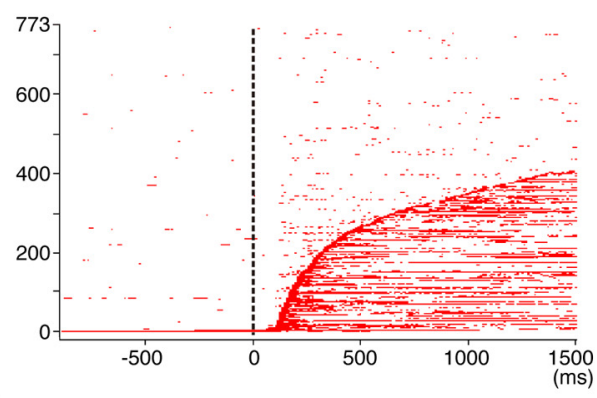

H Action (vIPFC)

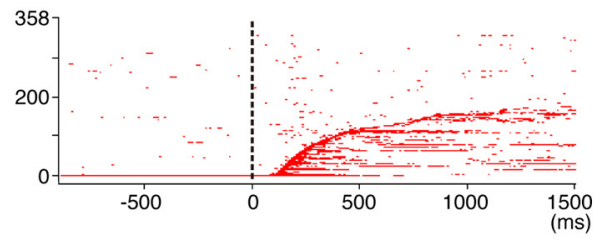

I Action (dIPFC)

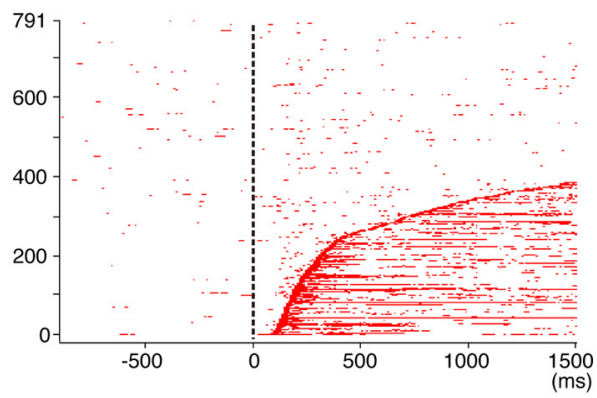

J Duration of selectivity (Action)

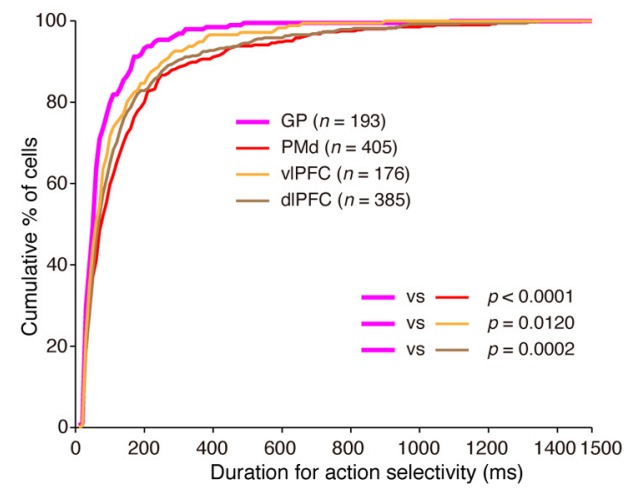

Figure 10. Temporal profiles of neuronal selectivity for behavioral goals and actions in the symbolic cue task. $A-D$, Time of appearance of goal selectivity in individual neurons in the GP ( $A$ ), PMd $(\boldsymbol{B}), \operatorname{VIPFC}(\boldsymbol{C})$, and dIPFC (D), plotted along the behavioral task period. The blue dots indicate the time points at which each neuron was selective for the goals. Each row (Figure legend continues.) 
In our previous studies, we found that goal representation in the PMd developed $60 \mathrm{~ms}$ later than the visuospatial representation (Yamagata et al., 2009). An intriguing possibility is that the slow development of goal representation is the result of BG involvement in the goal-retrieval process, whereas the more rapid development of visuospatial signals originates from direct inputs from the posterior parietal cortex to the PMd (Johnson et al., 1996; Matelli et al., 1998; Rozzi et al., 2006).

The task design of the present study did not allow us to distinguish neurons that integrate object features and goals (Asaad et al., 1998) from those that simply represent the object features themselves because both types of neurons were classified as object neurons. It also did not allow us to detect neurons that primarily represent goals and secondarily object features. These issues should be examined in the future.

\section{Action specification in the GP}

After the choice-cue presentation, GP neurons began to reflect information about the choice-cue location, mixed representation of the choice-cue location and behavioral goal, and action to be selected. However, among these neurons, those selective for a mixture of the choice-cue location and the behavioral goal were less frequent in the GP than in the PMd, indicating that the GP may not actively participate in the transformation process. The relatively late temporal development of action representation supports this view. In general, GP action representation develops $\sim 30 \mathrm{~ms}$ after the appearance in the PMd and lPFC. Furthermore, Muhammad et al. (2006) reported that behavioral responses tended to begin earlier in the PMd than in the striatum in a visuomotor task using the GO/NO-GO paradigm. Seo et al. (2012) revealed that the representation of the selected action occurred earlier in the IPFC than in the dorsal striatum. These observations suggest that, when action is determined based on visual signals, the action command is initially specified in cortical areas, such as the PMd and IPFC, and this, in turn, is followed by representation in the $\mathrm{BG}$.

\section{Timing and duration of behavioral-event representation in the GP compared with cortical areas: insights into neural computation}

By analyzing selectivity for excited and inhibited responses in the GP, we found that population selectivity for the object, visuospace, goal, and action developed 20-80 ms earlier in the excited responses than in the inhibited responses. Although the onsets of selectivity differed as a population, among individual neurons, the latencies of excited and inhibited neuronal responses did not differ significantly (Kolmogorov-Smirnov test, $p>0.05$; data

\footnotetext{
(Figure legend continued.) represents a neuron. Neurons were sorted according to the onset of goal selectivity after the instruction-cue onset, from bottom to top. $E$, Cumulative fractions of the duration of goal selectivity after the instruction cue onset in the GP (light blue), PMd (dark blue), vIPFC (dark purple), and dIPFC (light purple) in the symbolic cue task. $\boldsymbol{F}$-l, Time of appearance of action selectivity in the GP $(\boldsymbol{F}), \operatorname{PMd}(\boldsymbol{G}), \operatorname{vIPFC}(\boldsymbol{H})$, and dIPFC $(\boldsymbol{I})$. The red dots indicate the time points at which each neuron was selective for the actions. Each row represents a neuron. Neurons were sorted according to the onset of action selectivity after the choice-cue onset, from bottom to top. J, Cumulative fractions of the duration of action selectivity after the choice cue presentation in the GP (pink), PMd (red), vIPFC (orange), and dIPFC (brown) in the symbolic cue task. $\boldsymbol{E}$, J, The parentheses enclose the number of neurons with the onset of selectivity $\leq 2000 \mathrm{~ms}$ after the instruction-cue onset (for goal selectivity, $\boldsymbol{E}$ ) and $\leq 1500 \mathrm{~ms}$ after the choice-cue onset (for action selectivity, J). The $p$ values indicate the results of the statistical analysis conducted with these neurons (Kolmogorov-Smirnov test) examining differences between the GP and the three cortical areas.
}

not shown). These trends are consistent with previous reports that the latency of excited visual and saccade responses is shorter than that of inhibited responses (Kato and Hikosaka, 1995). By recording GP neurons while cortical motor areas were electrically stimulated, Nambu et al. (2000) revealed that the early excitatory responses in the GP, which begin $\sim 10 \mathrm{~ms}$ after stimulation, originate from the subthalamus. In contrast, inhibited responses, which begin $\sim 20 \mathrm{~ms}$ after stimulation, originate directly from the striatum. Thus, an intriguing possibility is that the hyperdirect pathway via the subthalamus plays an important role in the initial development of goal and action representations, as well as sensory responses in the GP.

We also found that goal and action representations by each GP neuron were transient in nature and much briefer than those in the PMd and dlPFC. Marsden (1982) proposed that "the basal ganglia might focus attention on a single event in the environment, to the exclusion of all others." Subsequently, Houk and Wise (1995) proposed that the BG plays a role in contextual pattern recognition. According to this theory, GP neurons transiently decrease or increase activity, giving rise to sustained activity enhancement (context registration) or suppression (context negation) in the thalamus and cerebral cortex. Our findings of transient GP neuron responses and the sustained responses of PMd and dlPFC neurons are in accord with these proposals. In the present study, after the instruction-cue onset, the onset of the activity reflecting visual objects and goals was comparable in the GP and in the vlPFC, dlPFC, and PMd, suggesting that the GP may provide a signal to inform wide cortical areas that a certain object or goal has appeared, serving to trigger subsequent information processing in these areas. After the choice-cue onset, action representation developed later in the GP than in the frontal cortex, suggesting that the GP may be involved in registering an established action, based on which competing motor programs are suppressed (Mink, 1996), or subsequent processes for action preparation and execution are initiated.

In contrast to the PMd and dlPFC, we found that the durations of goal selectivity of the vlPFC neurons were as short as those of GP neurons. This finding endorses the proposal that the vlPFC is not essential for maintaining working memory (Rushworth et al., 1997). Because vlPFC is amply interconnected with sensory association areas, it may play a role in registering sensory and relevant signals rather than maintaining them as memory signals. However, the functional relationships between the GP and vlPFC still remain elusive, calling for future investigations to reveal them.

In the present study, we did not detect any differences in GPe and GPi neurons with respect to the proportion of behavioralepoch selectivity or the timing of appearance of their response selectivity. In view of apparent differences in anatomical connectivity of GPe and GPi, it seems that we need new lines of research to reveal functional specializations in these areas.

\section{Sparse coding in the GP}

It appeared that the proportion of GP neurons representing each behavioral factor at individual time bins (200 ms) during the behavioral task in this study was rather modest (Fig. 4). On the other hand, we found that a majority of GP neurons studied ( $n=$ $695,69 \%$ of the total) exhibited selectivity to a component of behavioral factors at any time from the onset of instruction cue to the appearance of GO signal. Thus, the apparent modest numbers of selective neurons at each time interval are considered to be accounted for by the briefness of the duration of selectivity exhibited by individual neurons. By contrast, the magnitudes of 
selective responses representing each behavioral factor were of considerable degree: activity modulation of GP neurons amounted to $16-47$ spikes/s. The potency of neuronal responses is further characterized by the promptness of activity modulation as revealed by the population selectivity; the selectivity reached the peaks $170-350 \mathrm{~ms}$ after the cue onset. Together, the GP is viewed as coding information with a small number of strongly active neurons at any given point in time during the task. This type of information coding corresponds to the sparse coding, which is thought to be constituting crucial coding mechanisms in sensory (Olshausen and Field, 2004) and motor processing (Hahnloser et al., 2002). Our present observations suggest that the GP may use the sparse coding in determining behavioral goal and in specifying action. In the future, the sparse coding model might lead to a new perspective on information processing achieved by the cortico-basal ganglia networks.

In conclusion, we compared response properties of GP neurons with those of PMd and IPFC neurons in terms of behavioral goal determination and action specification. Our results indicate that the GP, along with the frontal areas, is involved in initial stages of goal determination based on visual signals. As for action specification, however, GP activity follows the leading activity in the PMd and IPFC. Together with the finding of shorter length of time representing goal and action than in the PMd and dlPFC, our observations point to a unique involvement of the GP in goal-directed behavior.

\section{References}

Alexander GE, Crutcher MD (1990) Functional architecture of basal ganglia circuits: neural substrates of parallel processing. Trends Neurosci 13:266271. CrossRef Medline

Alexander GE, DeLong MR, Strick PL (1986) Parallel organization of functionally segregated circuits linking basal ganglia and cortex. Annu Rev Neurosci 9:357-381. CrossRef Medline

Amiez C, Kostopoulos P, Champod AS, Petrides M (2006) Local morphology predicts functional organization of the dorsal premotor region in the human brain. J Neurosci 26:2724-2731. CrossRef Medline

Antzoulatos EG, Miller EK (2011) Differences between neural activity in prefrontal cortex and striatum during learning of novel abstract categories. Neuron 71:243-249. CrossRef Medline

Asaad WF, Rainer G, Miller EK (1998) Neural activity in the primate prefrontal cortex during associative learning. Neuron 21:1399-1407. CrossRef Medline

Brasted PJ, Wise SP (2004) Comparison of learning-related neuronal activity in the dorsal premotor cortex and striatum. Eur J Neurosci 19:721740. CrossRef Medline

Brown VJ, Desimone R, Mishkin M (1995) Responses of cells in the tail of the caudate nucleus during visual discrimination learning. J Neurophysiol 74:1083-1094. Medline

Caan W, Perrett DI, Rolls ET (1984) Responses of striatal neurons in the behaving monkey: 2 . Visual processing in the caudal neostriatum. Brain Res 290:53-65. CrossRef Medline

Cheng K, Saleem KS, Tanaka K (1997) Organization of corticostriatal and corticoamygdalar projections arising from the anterior inferotemporal area TE of the macaque monkey: a Phaseolus vulgaris leucoagglutinin study. J Neurosci 17:7902-7925. Medline

Cisek P, Kalaska JF (2010) Neural mechanisms for interacting with a world full of action choices. Annu Rev Neurosci 33:269-298. CrossRef Medline

Crucian GP, Okun MS (2003) Visual-spatial ability in Parkinson's disease. Front Biosci 8:s992-s997. CrossRef Medline

Crutcher MD, DeLong MR (1984) Single cell studies of the primate putamen: I. Functional organization. Exp Brain Res 53:233-243. CrossRef Medline

DeLong MR (1971) Activity of pallidal neurons during movement. J Neurophysiol 34:414-427. Medline

Dubois B, Pillon B (1997) Cognitive deficits in Parkinson's disease. J Neurol 244:2-8. Medline

Flaherty AW, Graybiel AM (1993) Output architecture of the primate putamen. J Neurosci 13:3222-3237. Medline
Flaherty AW, Graybiel AM (1994) Input-output organization of the sensorimotor striatum in the squirrel monkey. J Neurosci 14:599-610. Medline

Genovesio A, Tsujimoto S, Wise SP (2012) Encoding goals but not abstract magnitude in the primate prefrontal cortex. Neuron 74:656-662. CrossRef Medline

Giménez-Amaya JM, Graybiel AM (1990) Compartmental origins of the striatopallidal projection in the primate. Neuroscience 34:111-126. CrossRef Medline

Graybiel AM (2008) Habits, rituals, and the evaluative brain. Annu Rev Neurosci 31:359-387. CrossRef Medline

Graybiel AM, Aosaki T, Flaherty AW, Kimura M (1994) The basal ganglia and adaptive motor control. Science 265:1826-1831. CrossRef Medline

Hadj-Bouziane F, Boussaoud D (2003) Neuronal activity in the monkey striatum during conditional visuomotor learning. Exp Brain Res 153: 190-196. CrossRef Medline

Hahnloser RH, Kozhevnikov AA, Fee MS (2002) An ultra-sparse code underlies the generation of neural sequences in a songbird. Nature 419:6570. CrossRef Medline

Halsband U, Passingham RE (1985) Premotor cortex and the conditions for movement in monkeys (Macaca fascicularis). Behav Brain Res 18:269_ 277. CrossRef Medline

Hazrati LN, Parent A (1992) Differential patterns of arborization of striatal and subthalamic fibers in the two pallidal segments in primates. Brain Res 598:311-315. CrossRef Medline

Hollerman JR, Tremblay L, Schultz W (2000) Involvement of basal ganglia and orbitofrontal cortex in goal-directed behavior. Prog Brain Res 126: 193-215. CrossRef Medline

Hoshi E, Tanji J (2006) Differential involvement of neurons in the dorsal and ventral premotor cortex during processing of visual signals for action planning. J Neurophysiol 95:3596-3616. CrossRef Medline

Houk JC, Wise SP (1995) Distributed modular architectures linking basal ganglia, cerebellum, and cerebral cortex: their role in planning and controlling action. Cereb Cortex 5:95-110. CrossRef Medline

Inase M, Tanji J (1994) Projections from the globus pallidus to the thalamic areas projecting to the dorsal area 6 of the macaque monkey: a multiple tracing study. Neurosci Lett 180:135-137. CrossRef Medline

Inase M, Li BM, Takashima I, Ijijima T (2001) Pallidal activity is involved in visuomotor association learning in monkeys. Eur J Neurosci 14:897-901. CrossRef Medline

Johnson PB, Ferraina S, Bianchi L, Caminiti R (1996) Cortical networks for visual reaching: physiological and anatomical organization of frontal and parietal lobe arm regions. Cereb Cortex 6:102-119. CrossRef Medline

Kaneda K, Nambu A, Tokuno H, Takada M (2002) Differential processing patterns of motor information via striatopallidal and striatonigral projections. J Neurophysiol 88:1420-1432. Medline

Kato M, Hikosaka O (1995) Function of the indirect pathway in the basal ganglia oculomotor system: visuo-oculomotor activities of external pallidum neurons. In: Age-related dopamine-dependent disorders (Monographs in neural sciences) (Segawa M, Nomura Y, eds). Basel: Karger, pp $178-187$.

Kita H, Nambu A, Kaneda K, Tachibana Y, Takada M (2004) Role of ionotropic glutamatergic and GABAergic inputs on the firing activity of neurons in the external pallidum in awake monkeys. J Neurophysiol 92:3069-3084. CrossRef Medline

Kurata K, Hoffman DS (1994) Differential effects of muscimol microinjection into dorsal and ventral aspects of the premotor cortex of monkeys. J Neurophysiol 71:1151-1164. Medline

Marsden CD (1982) The mysterious motor function of the basal ganglia: the Robert Wartenberg Lecture. Neurology 32:514-539. CrossRef Medline

Matelli M, Govoni P, Galletti C, Kutz DF, Luppino G (1998) Superior area 6 afferents from the superior parietal lobule in the macaque monkey. J Comp Neurol 402:327-352. CrossRef Medline

Maurice N, Deniau JM, Glowinski J, Thierry AM (1998) Relationships between the prefrontal cortex and the basal ganglia in the rat: physiology of the corticosubthalamic circuits. J Neurosci 18:9539-9546. Medline

Maurice N, Deniau JM, Glowinski J, Thierry AM (1999) Relationships between the prefrontal cortex and the basal ganglia in the rat: physiology of the cortico-nigral circuits. J Neurosci 19:4674-4681. Medline

Mendez MF, Adams NL, Lewandowski KS (1989) Neurobehavioral changes associated with caudate lesions. Neurology 39:349-354. CrossRef Medline 
Middleton FA, Strick PL (1994) Anatomical evidence for cerebellar and basal ganglia involvement in higher cognitive function. Science 266:458461. CrossRef Medline

Middleton FA, Strick PL (2000) Basal ganglia and cerebellar loops: motor and cognitive circuits. Brain Res Brain Res Rev 31:236-250. CrossRef Medline

Middleton FA, Strick PL (2002) Basal-ganglia "projections" to the prefrontal cortex of the primate. Cereb Cortex 12:926-935. CrossRef Medline

Mink JW (1996) The basal ganglia: focused selection and inhibition of competing motor programs. Prog Neurobiol 50:381-425. CrossRef Medline

Mink JW, Thach WT (1993) Basal ganglia intrinsic circuits and their role in behavior. Curr Opin Neurobiol 3:950-957. CrossRef Medline

Muhammad R, Wallis JD, Miller EK (2006) A comparison of abstract rules in the prefrontal cortex, premotor cortex, inferior temporal cortex, and striatum. J Cogn Neurosci 18:974-989. CrossRef Medline

Nakayama Y, Yamagata T, Tanji J, Hoshi E (2008) Transformation of a virtual action plan into a motor plan in the premotor cortex. J Neurosci 28:10287-10297. CrossRef Medline

Nambu A, Tokuno H, Hamada I, Kita H, Imanishi M, Akazawa T, Ikeuchi Y, Hasegawa N (2000) Excitatory cortical inputs to pallidal neurons via the subthalamic nucleus in the monkey. J Neurophysiol 84:289-300. Medline

Nixon PD, McDonald KR, Gough PM, Alexander IH, Passingham RE (2004) Cortico-basal ganglia pathways are essential for the recall of wellestablished visuomotor associations. Eur J Neurosci 20:3165-3178. CrossRef Medline

Olshausen BA, Field DJ (2004) Sparse coding of sensory inputs. Curr Opin Neurobiol 14:481-487. CrossRef Medline

Scalaidhe SP, Wilson FA, Goldman-Rakic PS (1997) Areal segregation of face-processing neurons in prefrontal cortex. Science 278:1135-1138. CrossRef Medline

Packard MG, Knowlton BJ (2002) Learning and memory functions of the basal ganglia. Annu Rev Neurosci 25:563-593. CrossRef Medline

Parent A, Hazrati LN (1995a) Functional anatomy of the basal ganglia: II. The place of subthalamic nucleus and external pallidum in basal ganglia circuitry. Brain Res Brain Res Rev 20:128-154. CrossRef Medline

Parent A, Hazrati LN (1995b) Functional anatomy of the basal ganglia: I. The cortico-basal ganglia-thalamo-cortical loop. Brain Res Brain Res Rev 20:91-127. CrossRef Medline

Pasupathy A, Miller EK (2005) Different time courses of learning-related activity in the prefrontal cortex and striatum. Nature 433:873-876. CrossRef Medline

Petrides M (1986) The effect of periarcuate lesions in the monkey on the performance of symmetrically and asymmetrically reinforced visual and auditory go, no-go tasks. J Neurosci 6:2054-2063. Medline

Rozzi S, Calzavara R, Belmalih A, Borra E, Gregoriou GG, Matelli M, Luppino G (2006) Cortical connections of the inferior parietal cortical convexity of the macaque monkey. Cereb Cortex 16:1389-1417. CrossRef Medline

Rushworth MF, Nixon PD, Eacott MJ, Passingham RE (1997) Ventral prefrontal cortex is not essential for working memory. J Neurosci 17:48294838. Medline

Ryan LJ, Sanders DJ (1994) Subthalamic nucleus and globus pallidus lesions alter activity in nigrothalamic neurons in rats. Brain Res Bull 34:19-26. CrossRef Medline

Saga Y, Hirata Y, Takahara D, Inoue K, Miyachi S, Nambu A, Tanji J, Takada
M, Hoshi E (2011) Origins of multisynaptic projections from the basal ganglia to rostrocaudally distinct sectors of the dorsal premotor area in macaques. Eur J Neurosci 33:285-297. CrossRef Medline

Saint-Cyr JA, Ungerleider LG, Desimone R (1990) Organization of visual cortical inputs to the striatum and subsequent outputs to the pallidonigral complex in the monkey. J Comp Neurol 298:129-156. CrossRef Medline

Selemon LD, Goldman-Rakic PS (1985) Longitudinal topography and interdigitation of corticostriatal projections in the rhesus monkey. J Neurosci 5:776-794. Medline

Seo M, Lee E, Averbeck BB (2012) Action selection and action value in frontal-striatal circuits. Neuron 74:947-960. CrossRef Medline

Shin S, Sommer MA (2010) Activity of neurons in monkey globus pallidus during oculomotor behavior compared with that in substantia nigra pars reticulata. J Neurophysiol 103:1874-1887. CrossRef Medline

Tachibana Y, Kita H, Chiken S, Takada M, Nambu A (2008) Motor cortical control of internal pallidal activity through glutamatergic and GABAergic inputs in awake monkeys. Eur J Neurosci 27:238-253. CrossRef Medline

Toni I, Rushworth MF, Passingham RE (2001) Neural correlates of visuomotor associations: spatial rules compared with arbitrary rules. Exp Brain Res 141:359-369. CrossRef Medline

Toni I, Rowe J, Stephan KE, Passingham RE (2002) Changes of corticostriatal effective connectivity during visuomotor learning. Cereb Cortex 12:1040-1047. CrossRef Medline

Uc EY, Rizzo M, Anderson SW, Qian S, Rodnitzky RL, Dawson JD (2005) Visual dysfunction in Parkinson disease without dementia. Neurology 65:1907-1913. CrossRef Medline

Wallis JD, Miller EK (2003) From rule to response: neuronal processes in the premotor and prefrontal cortex. J Neurophysiol 90:1790-1806. CrossRef Medline

Wang M, Zhang H, Li BM (2000) Deficit in conditional visuomotor learning by local infusion of bicuculline into the ventral prefrontal cortex in monkeys. Eur J Neurosci 12:3787-3796. CrossRef Medline

Webster MJ, Bachevalier J, Ungerleider LG (1993) Subcortical connections of inferior temporal areas TE and TEO in macaque monkeys. J Comp Neurol 335:73-91. CrossRef Medline

Wise SP, Murray EA (2000) Arbitrary associations between antecedents and actions. Trends Neurosci 23:271-276. CrossRef Medline

Wise SP, Murray EA, Gerfen CR (1996) The frontal cortex-basal ganglia system in primates. Crit Rev Neurobiol 10:317-356. CrossRef Medline

Yamagata T, Nakayama Y, Tanji J, Hoshi E (2009) Processing of visual signals for direct specification of motor targets and for conceptual representation of action targets in the dorsal and ventral premotor cortex. J Neurophysiol 102:3280-3294. CrossRef Medline

Yamagata T, Nakayama Y, Tanji J, Hoshi E (2012) Distinct information representation and processing for goal-directed behavior in the dorsolateral and ventrolateral prefrontal cortex and the dorsal premotor cortex. J Neurosci 32:12934-12949. CrossRef Medline

Yamamoto S, Monosov IE, Yasuda M, Hikosaka O (2012) What and where information in the caudate tail guides saccades to visual objects. J Neurosci 32:11005-11016. CrossRef Medline

Yoshida A, Tanaka M (2009) Enhanced modulation of neuronal activity during antisaccades in the primate globus pallidus. Cereb Cortex 19:206217. CrossRef Medline 